\title{
Error Bounds for the Method of Simultaneous Projections with Infinitely Many Subspaces
}

\author{
Simeon Reich* $\quad$ Rafał Zalas ${ }^{\dagger}$
}

August 31, 2021

\begin{abstract}
We investigate the properties of the simultaneous projection method as applied to countably infinitely many closed and linear subspaces of a real Hilbert space. We establish the optimal error bound for linear convergence of this method, which we express in terms of the cosine of the Friedrichs angle computed in an infinite product space. In addition, we provide estimates and alternative expressions for the above-mentioned number. Furthermore, we relate this number to the dichotomy theorem and to super-polynomially fast convergence. We also discuss polynomial convergence of the simultaneous projection method which takes place for particularly chosen starting points.
\end{abstract}

Key words and phrases: Friedrichs angle; Product space; Rates of convergence; Simultaneous projection method

2010 Mathematics Subject Classification: 41A25, 41A28, 41A44, 41A65.

\section{Introduction}

Let $\mathcal{H}$ be a real Hilbert space with its inner product denoted by $\langle\cdot, \cdot\rangle$ and the induced norm denoted by $\|\cdot\|$. In this paper we study the asymptotic properties of the simultaneous projection method as applied to a possibly countably infinite number of closed and linear subspaces of $\mathcal{H}$. We begin by briefly recalling some of the known results which have so far been established only for a finite number of subspaces. Moreover, we recall relevant results related to the cyclic projection method. We do not discuss here the case of general closed and convex sets, for which we refer the interested reader to [9, 11, 13, 14, 15]. For other examples of projection methods studied in the setting of closed and linear subspaces, we refer the reader to $[1,5,6,7,34]$.

\subsection{Related Work}

For now let $r \in \mathbb{Z}_{+}$. For each $i=1,2, \ldots, r$, let $M_{i} \subset \mathcal{H}$ be a nontrivial closed and linear subspace, and let $P_{M_{i}}$ denote the corresponding orthogonal projection. Moreover, let $M:=\bigcap_{i=1}^{r} M_{i}$ with the corresponding orthogonal projection $P_{M}$. In the next three theorems, the operator $T_{r}$ can be either the cyclic projection operator $T_{r}:=P_{M_{r}} \ldots P_{M_{1}}$ or the simultaneous projection operator $T_{r}:=\frac{1}{r} \sum_{i=1}^{r} P_{M_{i}}$. In particular, $T_{2}=P_{M_{2}} P_{M_{1}}$ is the alternating projection operator. We begin with a well-known result.

\footnotetext{
*Department of Mathematics, The Technion - Israel Institute of Technology, 3200003 Haifa, Israel; E-mail: sreich@technion.ac.il.

${ }^{\dagger}$ Department of Mathematics, The Technion - Israel Institute of Technology, 3200003 Haifa, Israel; E-mail: zalasrafal@gmail.com.
} 
Theorem 1.1 (Norm Convergence). For each $x \in \mathcal{H}$, we have

$$
\lim _{k \rightarrow \infty}\left\|T_{r}^{k}(x)-P_{M}(x)\right\|=0 .
$$

Theorem 1.1 goes back to von Neumann [36], when $T_{2}=P_{M_{2}} P_{M_{1}}$, and to Halperin [22], when $T_{r}=P_{M_{r}} \ldots P_{M_{1}}$. Lapidus [27] and Reich [32] proved Theorem 1.1 for $T_{r}=\frac{1}{r} \sum_{i=1}^{r} P_{M_{i}}$.

It turns out that in the infinite dimensional case, the convergence properties can indeed differ from their finite dimensional counterparts.

Theorem 1.2 (Dichotomy). Exactly one of the following two statements holds:

(i) $\sum_{i=1}^{r} M_{i}^{\perp}$ is closed. Then the sequence $\left\{T_{r}^{k}\right\}_{k=1}^{\infty}$ converges linearly to $P_{M}$.

(ii) $\sum_{i=1}^{r} M_{i}^{\perp}$ is not closed. Then the sequence $\left\{T_{r}^{k}\right\}_{k=1}^{\infty}$ converges arbitrarily slowly to $P_{M}$.

We recall that the linear convergence in (i) means that there are constants $c>0$ and $q \in(0,1)$ such that the inequality $\left\|T_{r}^{k}(x)-P_{M}(x)\right\| \leq c q^{k}\|x\|$ holds for all $k=1,2, \ldots$ and all $x \in \mathcal{H}$. The arbitrarily slow convergence in (ii) means that for any sequence of scalars $\left\{a_{k}\right\}_{k=1}^{\infty}$ with $0 \leq a_{k}$ and $a_{k} \rightarrow 0$, there is a point $x \in \mathcal{H}$ such that the inequality $\left\|T_{r}(x)-P_{M}(x)\right\| \geq a_{k}$ holds for all $k=1,2, \ldots$

The first instance of Theorem 1.2 (ii) is due to Bauschke, Deutsch and Hundal [10], who proved it for the alternating projection method $\left(T_{2}=P_{M_{2}} P_{M_{1}}\right)$ with decreasing null sequences $\left\{a_{k}\right\}_{k=1}^{\infty}$. These authors commented that their result is also valid for $T_{r}=\frac{1}{r} \sum_{i=1}^{r} P_{M_{i}}$ with $r \geq 2$ because of the connection between the method of simultaneous projections and the method of alternating projections in the product space. We return to this connection below. The statement of Theorem 1.2 with $T_{r}=P_{M_{r}} \ldots P_{M_{1}}$, allowing $r \geq 2$ and any null nonnegative sequence, has been established by Deutsch and Hundal in [18]. Similar results can be found, for example, in [3, 4, 5, 19, 20].

Despite the arbitrarily slow convergence presented in alternative (ii), there do exist sets of starting points in $\mathcal{H}$ for which there are relatively good error upper bounds. We comment on this matter in Theorems 1.3 and 1.4 .

Theorem 1.3 (Super-polynomial Rate). If $\sum_{i=1}^{r} M_{i}^{\perp}$ is not closed, then the sequence $\left\{T_{r}^{k}\right\}_{k=1}^{\infty}$ converges super-polynomially fast to $P_{M}$ on some dense linear subspace $X \subset \mathcal{H}$.

The super-polynomially fast convergence means that $k^{n}\left\|T_{r}^{k}(x)-P_{M}(x)\right\| \rightarrow 0$ as $k \rightarrow \infty$ for each $x \in X$ and for all $n=1,2, \ldots$. Theorem 1.3 is due to Badea and Seifert [4], who established it for $T_{r}:=P_{M_{r}} \ldots P_{M_{1}}$ in a complex Hilbert space. By using a complexification argument, we see that this result is also valid in a real Hilbert space. Similarly to the case of Theorem 1.2, the result holds for $T_{r}=\frac{1}{r} \sum_{i=1}^{r} P_{M_{i}}$ as can be seen by using the product space approach. The details can be found in [33].

The following theorem has recently been established by Borodin and Kopecká in [12].

Theorem 1.4 (Polynomial Rate). Assume that $M_{1} \cap M_{2}=\{0\}$. Then for any $x \in M_{1}^{\perp}+M_{2}^{\perp}$ there is $C(x)>0$ such that

$$
\left\|\left(P_{M_{2}} P_{M_{1}}\right)^{k}(x)\right\| \leq \frac{C(x)}{\sqrt{k}}, \quad k=1,2, \ldots
$$

Moreover, when $\mathcal{H}$ is infinite dimensional, the denominator $\sqrt{k}$ cannot be replaced by $k^{1 / 2+\varepsilon}$ for any $\varepsilon>0$ (that is, for each $\varepsilon>0$ there are two closed linear subspaces $M_{1}, M_{2}$ and $x \in M_{1}^{\perp}+M_{2}^{\perp}$ such that $\left\|\left(P_{M_{2}} P_{M_{1}}\right)^{k}(x)\right\| \geq C(x) k^{-(1 / 2+\varepsilon)}$ for some $C(x)>0$ and all $\left.k=1,2, \ldots\right)$. 
It is not difficult to see that estimate (1.2) also holds when $M_{1}^{\perp} \cap M_{2}^{\perp} \neq\{0\}$. We comment on this in the proof of Theorem 5.4 below.

We now return to the case where $\sum_{i=1}^{r} M_{i}^{\perp}$ is closed. In this case one may be interested in finding the optimal error bound, that is, the smallest possible estimate for the relative error $e_{k}(x):=\| T_{r}^{k}(x)-$ $P_{M}(x)\|/\| x \|$, which is independent of $x$. The answer to this question leads to the computation of the operator norm since $\sup _{x \neq 0} e_{k}(x)=\left\|T_{r}^{k}-P_{M}\right\|$.

The first result of this type for the alternating projections method (APM) is due to Aronszajn (inequality) [2], and Kayalar and Weinert (equality) [25], who expressed the optimal error bound in terms of the cosine of the Friedrichs angle between the subspaces $M_{1}$ and $M_{2}$, which we denote by $\cos \left(M_{1}, M_{2}\right)$. Recall that

$$
\cos \left(M_{1}, M_{2}\right):=\sup \left\{\langle x, y\rangle: \begin{array}{l}
x \in M_{1} \cap\left(M_{1} \cap M_{2}\right)^{\perp} \cap B, \\
y \in M_{2} \cap\left(M_{1} \cap M_{2}\right)^{\perp} \cap B
\end{array}\right\} \in[0,1],
$$

where $B:=\{x \in \mathcal{H}:\|x\| \leq 1\}$. Their result reads as follows:

Theorem 1.5 (Optimal Error Bound). For each $k=1,2, \ldots$, we have

$$
\left\|\left(P_{M_{2}} P_{M_{1}}\right)^{k}-P_{M}\right\|=\cos \left(M_{1}, M_{2}\right)^{2 k-1} .
$$

Only estimates are known for $r>2$; see, for example, $[25,30]$ for those which involve angles measured between $M_{1} \cap \ldots \cap M_{i}$ and $M_{i+1}, i=1, \ldots, r-1$, and [3, 31] for those which are expressed using the so-called inclination number. At this point, recall that

$$
\cos \left(M_{1}, M_{2}\right)<1 \quad \Longleftrightarrow \quad M_{1}^{\perp}+M_{2}^{\perp} \text { is closed; }
$$

see, for example, $[16,8]$ and $[17$, Theorem 9.35 and p. 235] for detailed historical notes. Equivalence (1.5) may also be deduced from Theorems 1.2 and 1.5.

A result analogous to Theorem 1.5 has been established in [33] for the simultaneous projection method. Indeed, let the product space $\mathbf{H}_{r}:=\mathcal{H}^{r}=\mathcal{H} \times \ldots \times \mathcal{H}$ be equipped with the inner product $\langle\mathbf{x}, \mathbf{y}\rangle_{r}:=$ $\frac{1}{r} \sum_{i=1}^{r}\left\langle x_{i}, y_{i}\right\rangle$ and the induced norm $\|\mathbf{x}\|_{r}:=\sqrt{\langle\mathbf{x}, \mathbf{x}\rangle_{r}}$, where $\mathbf{x}=\left\{x_{1}, \ldots, x_{r}\right\}, \mathbf{y}=\left\{y_{1}, \ldots, y_{r}\right\}$. Moreover, let $\mathbf{C}_{r}:=M_{1} \times \ldots \times M_{r} \subset \mathbf{H}_{r}$ and $\mathbf{D}_{r}:=\{\{x, \ldots, x\}: x \in \mathcal{H}\} \subset \mathbf{H}_{r}$, and denote by $\cos _{r}\left(\mathbf{C}_{r}, \mathbf{D}_{r}\right)$ the corresponding cosine of the Friedrichs angle in $\mathbf{H}_{r}$; see (2.9) and Remark 2.7.

Theorem 1.6 (Optimal Error Bound). For each $k=1,2, \ldots$, we have

$$
\left\|\left(\frac{1}{r} \sum_{i=1}^{r} P_{M_{i}}\right)^{k}-P_{M}\right\|=\cos _{r}\left(\mathbf{C}_{r}, \mathbf{D}_{r}\right)^{2 k} .
$$

In particular, when $r=2$, we get $\cos _{2}\left(\mathbf{C}_{2}, \mathbf{D}_{2}\right)^{2}=\frac{1}{2}+\frac{1}{2} \cos \left(M_{1}, M_{2}\right)$.

Recall that the alternating projection formalization introduced above is due to Pierra [29], who observed that for each $x \in \mathcal{H}, \mathbf{x}=(x, \ldots, x)$ and $k=1,2, \ldots$, we have $\left\|\left(\frac{1}{r} \sum_{i=1}^{r} P_{M_{i}}\right)^{k}(x)-P_{M}(x)\right\|=$ $\left\|\left(P_{\mathbf{D}_{r}} P_{\mathbf{C}_{r}}\right)^{k}(\mathbf{x})-P_{\mathbf{C}_{r} \cap \mathbf{D}_{r}}(\mathbf{x})\right\|_{r}$. Note here that by simply combining this with Theorem 1.5, we only obtain an upper bound given by $\cos _{r}\left(\mathbf{C}_{r}, \mathbf{D}_{r}\right)^{2 k-1}$. The properties of the $\operatorname{cosine} \cos _{r}\left(\mathbf{C}_{r}, \mathbf{D}_{r}\right)$ were studied in [3], where equality (1.6) was shown for $k=1$.

It turns out that when $r=2$, the cosine of the Friedrichs angle appears in the optimal rate estimates for many other well-known projection methods. See, for example, [7] for the relaxed alternating projection 
method, [6] for the Douglas-Rachford method or [1] for the method of averaged alternating modified reflections. We refer the interested reader to [1, Table 1], where one can find an elegant comparison of rates.

\subsection{Contribution and Organization of the Paper}

The purpose of the present paper is to investigate the asymptotic properties of the simultaneous projection operator, analogous to those mentioned above, in the case where the number of subspaces $M_{i}$ is possibly countably infinite, that is, when $r \in \mathbb{Z}_{+} \cup\{\infty\}$. The aforesaid operator is defined by $T_{\omega}:=\sum_{i=1}^{r} \omega_{i} P_{M_{i}}$, where $\omega=\left\{\omega_{i}\right\}_{i=1}^{r}$ is a vector/sequence of weights $\omega_{i} \in(0,1)$ the sum of which equals one.

We carry out our study by adjusting the product space formalization of Pierra. For this purpose, for each operator $T_{\omega}$, we define the weighted product space $\left(\mathbf{H}_{\omega},\langle\cdot, \cdot\rangle_{\omega}\right)$, which is the analogue of $\left(\mathbf{H}_{r},\langle\cdot, \cdot\rangle_{r}\right)$, the subspaces $\mathbf{C}_{\omega}$ and $\mathbf{D}_{\omega}$ in $\mathbf{H}_{\omega}$, which correspond to $\mathbf{C}_{r}$ and $\mathbf{D}_{r}$ in $\mathbf{H}_{r}$ and finally, the cosine of the Friedrichs angle $\cos _{\omega}\left(\mathbf{C}_{\omega}, \mathbf{D}_{\omega}\right)$, which is an analogue of $\cos _{r}\left(\mathbf{C}_{r}, \mathbf{D}_{r}\right)$; see Section 2 for fully-fledged definitions, notation and basic properties. We note here only that for $r=\infty$ the product space $\mathbf{H}_{\omega}$ coincides with $\ell_{\omega}^{2}(\mathcal{H}):=\left\{\mathbf{x}=\left\{x_{i}\right\}_{i=1}^{\infty}: x_{i} \in \mathcal{H}, i=1,2, \ldots, \sum_{i=1}^{\infty} \omega_{i}\left\|x_{i}\right\|^{2}<\infty\right\}$.

We begin this study in Section 3 by showing the explicit connection between the operator $T_{\omega}$, the projection onto $M$ and the projections onto $\mathbf{C}_{\omega}, \mathbf{D}_{\omega}$ and $\mathbf{C}_{\omega} \cap \mathbf{D}_{\omega}$. Within this framework, we establish that the iterates of the simultaneous projection method $\left\{T_{\omega}^{k}(x)\right\}_{k=1}^{\infty}$ converge in norm to $P_{M}(x)$ for each starting point $x \in \mathcal{H}$, even when $r=\infty$. Moreover, using the powers of $\cos _{\omega}\left(\mathbf{C}_{\omega}, \mathbf{D}_{\omega}\right)^{2}$, we find an expression for the norm $\left\|T_{\omega}^{k}-P_{M}\right\|$, which, when smaller than 1, becomes the optimal error bound for linear convergence.

In Section 4 we present a detailed study of the $\operatorname{cosine} \cos _{\omega}\left(\mathbf{C}_{\omega}, \mathbf{D}_{\omega}\right)$. In particular, we provide an alternative formula for it, where the supremum is taken over a possibly smaller set (Lemma 4.1). Furthermore, we find a new estimate, which, depending on the weights $\omega$, may hold as a strict inequality, or as an equality (see Lemma 4.3 and Example 4.8). The important property of this estimate is that it must hold as an equality whenever the subspaces $\mathbf{C}_{\omega}$ and $\mathbf{D}_{\omega}$ are parallel and in this case the equality holds for all weights $\omega$ (Theorem 4.9). On the other hand, we show that the cosine can be easily evaluated when the subspaces $M_{i}$ are pairwise orthogonal (Proposition 4.7). In addition, we point out that by reducing the multiple copies of the subspaces $M_{i}$, the $\operatorname{cosine} \cos _{\omega}\left(\mathbf{C}_{\omega}, \mathbf{D}_{\omega}\right)$ can be computed in a simpler manner. For this reason we introduce a rearrangement lemma (see the Appendix). On the other hand, when $r=\infty$, we can approximate the $\operatorname{cosine}^{\cos \omega}\left(\mathbf{C}_{\omega}, \mathbf{D}_{\omega}\right)$ by a limit process of cosines between $\mathbf{C}_{q}$ and $\mathbf{D}_{q}$ defined in smaller product spaces, where $q \in \mathbb{Z}_{+}$and $q \rightarrow \infty$.

In Section 5 we return to the asymptotic properties of the simultaneous projection method. Building on the idea of $\ell^{2}$-summability, we replace the subspace $\sum_{i=1}^{r} M_{i}^{\perp}$, which plays a central role in Theorems 1.2-1.4, by another $\omega$-dependent subspace, which for $r=\infty$ becomes $\left\{\sum_{i=1}^{\infty} \omega_{i} x_{i}: x_{i} \in M_{i}^{\perp}, i=\right.$ $1,2, \ldots, \sum_{i=1}^{\infty} \omega_{i}\left\|x_{i}\right\|^{2}<\infty$; ; see (2.23). We show that this subspace, which we denote by $A_{\omega}\left(\mathbf{C}_{\omega}^{\perp}\right)$, plays a similar role to that of $\sum_{i=1}^{r} M_{i}^{\perp}$. In particular, the closedness of this subspace, or lack thereof, determines the dichotomy between linear and arbitrarily slow convergence. The latter case also implies the super-polynomially fast convergence on some dense linear subspace of $\mathcal{H}$. Moreover, $A_{\omega}\left(\mathbf{C}_{\omega}^{\perp}\right)$ becomes the set of "good" starting points on which we always have at least a polynomial rate of convergence.

It is not difficult to see, that when $r=\infty$, the sets $A_{\omega}\left(\mathbf{C}_{\omega}^{\perp}\right)$ may differ for different sequences of 
weights $\omega$; see Example 2.6. In spite of this, we find that the closedness of $A_{\omega}\left(\mathbf{C}_{\omega}^{\perp}\right)$ in $\mathcal{H}$ does not depend on the weights $\omega$, but only on the subspaces $M_{i}$ themselves. To be more precise, we prove that if the set $A_{\omega}\left(\mathbf{C}_{\omega}^{\perp}\right)$ is closed for one sequence of weights $\omega$, then it must be closed for all sequences of weights and be equal to $M^{\perp}$ (see Theorem 4.9 in Section 4 phrased in the language of cosines and Proposition 2.5). Hence it cannot happen that the rate of convergence is linear for one sequence $\omega$, but is arbitrarily slow for another one. This observation slightly strengthens the aforementioned dichotomy theorem.

\section{Preliminaries}

From now on, let

$$
r \in \mathbb{Z}_{+} \cup\{\infty\}
$$

and let

$$
\Omega_{r}:=\left\{\left\{\omega_{i}\right\}_{i=1}^{r}: \omega_{i}>0, i=1, \ldots, r, \sum_{i=1}^{r} \omega_{i}=1\right\} .
$$

In this section we extend the notation used in the introduction for the particular vector $\omega=\{1 / r, \ldots, 1 / r\} \in$ $\Omega_{r}$ to an arbitrary vector $\omega \in \Omega_{r}$, when $r \in \mathbb{Z}_{+}$, and to an arbitrary sequence $\omega \in \Omega_{\infty}$, when $r=\infty$.

For each $\omega \in \Omega_{r}$, we define a weighted product $\ell^{2}$-space and an associated weighted inner product by

$$
\mathbf{H}_{\omega}:=\left\{\begin{array}{ll}
\mathcal{H}^{r}, & \text { if } r \in \mathbb{Z}_{+}, \\
\ell_{\omega}^{2}(\mathcal{H}), & \text { if } r=\infty
\end{array} \quad \text { and } \quad\langle\mathbf{x}, \mathbf{y}\rangle_{\omega}:=\sum_{i=1}^{r} \omega_{i}\left\langle x_{i}, y_{i}\right\rangle,\right.
$$

where $\ell_{\omega}^{2}(\mathcal{H}):=\left\{\mathbf{x}=\left\{x_{i}\right\}_{i=1}^{\infty}: x_{i} \in \mathcal{H}, i=1,2, \ldots, \sum_{i=1}^{\infty} \omega_{i}\left\|x_{i}\right\|^{2}<\infty\right\}$ and where $\mathbf{x}=\left\{x_{i}\right\}_{i=1}^{r}, \mathbf{y}=$ $\left\{y_{i}\right\}_{i=1}^{r} \in \mathbf{H}_{\omega}$. One can verify that the pair $\left(\mathbf{H}_{\omega},\langle\cdot, \cdot\rangle_{\omega}\right)$ is a Hilbert space. The induced norm on $\mathbf{H}_{\omega}$ and the operator norm on $\mathcal{B}\left(\mathbf{H}_{\omega}\right)$, the Banach space of all bounded linear operators on $\mathbf{H}_{\omega}$, are both denoted by $\|\cdot\|_{\omega}$. Notice that, when $r=\infty$, the weighted $\ell^{2}$-spaces $\mathbf{H}_{\omega}$ may be different for different $\omega \in \Omega_{\infty}$.

Example 2.1 $(r=\infty)$. Let $\alpha>0$ and $\beta>1$. Consider the weighted $\ell^{2}$-space $\mathbf{H}_{\omega, \beta}=\ell_{\omega, \beta}^{2}(\mathcal{H})$ with weights $\omega_{i, \beta}:=1 /\left(i^{\beta} s_{\beta}\right)$, where $s_{\beta}:=\sum_{i=1}^{\infty} 1 / i^{\beta}$. Moreover, let $\mathbf{x}_{\alpha}=\left\{x_{i}\right\}_{i=1}^{\infty}$ be any sequence with $\left\|x_{i}\right\|=i^{\alpha / 2}$. Then $\mathbf{x}_{\alpha} \in \mathbf{H}_{\omega, \beta}$ if and only if $\beta>1+\alpha$. Consequently, for any fixed $\alpha>0$, we can find $\beta \neq \beta^{\prime}$ (for example, $1<\beta^{\prime}<1+\alpha<\beta$ ) such that $\mathbf{x}_{\alpha} \in \mathbf{H}_{\omega, \beta}$, but $\mathbf{x}_{\alpha} \notin \mathbf{H}_{\omega, \beta^{\prime}}$.

For each $\omega \in \Omega_{r}$, we define the averaging operator $A_{\omega}: \mathbf{H}_{\omega} \rightarrow \mathcal{H}$ by

$$
A_{\omega}(\mathbf{x}):=\sum_{i=1}^{r} \omega_{i} x_{i}
$$

where $\mathbf{x}=\left\{x_{i}\right\}_{i=1}^{r} \in \mathbf{H}_{\omega}$. Note that $A_{\omega}$ is well defined for $r=\infty$, as the following proposition shows.

Proposition $2.2(r=\infty)$. If $\mathbf{x}=\left\{x_{i}\right\}_{i=1}^{\infty} \in \mathbf{H}_{\omega}$ for some $\omega \in \Omega_{\infty}$, then the series $\sum_{i=1}^{\infty} \omega_{i} x_{i}$ is absolutely convergent, hence unconditionally (compare with the Appendix).

Proof. Observe that $\omega_{i}\left\|x_{i}\right\|<\omega_{i}$ for all $i \in I:=\left\{i:\left\|x_{i}\right\|<1\right\}$ and $\omega_{j}\left\|x_{j}\right\| \leq \omega_{j}\left\|x_{j}\right\|^{2}$ for all $j \in J:=$ $\left\{j:\left\|x_{j}\right\| \geq 1\right\}$. Consequently, for each $n \geq 1$, we get

$$
\sum_{i=1}^{n}\left\|\omega_{i} x_{i}\right\|=\sum_{\substack{1 \leq i \leq n \\ i \in \bar{I}}} \omega_{i}\left\|x_{i}\right\|+\sum_{\substack{1 \leq j \leq n \\ j \in J}} \omega_{j}\left\|x_{j}\right\| \leq \sum_{\substack{1 \leq i \leq n \\ i \in \bar{I}}} \omega_{i}+\sum_{\substack{1 \leq j \leq n \\ j \in J}} \omega_{j}\left\|x_{j}\right\|^{2} \leq 1+\|\mathbf{x}\|_{\omega}^{2}<\infty,
$$

with the convention that the summation over the empty set is zero. 
In particular, the unconditional convergence of $A_{\omega}(\mathbf{x})$ for $r=\infty$ gives us a lot of freedom in rearranging the summands in (2.4); see Lemma 6.1 in the Appendix. Moreover, $A_{\omega}$ is a norm one linear operator which for all $\mathbf{x} \in \mathbf{H}_{\omega}$ and $z \in \mathcal{H}$ satisfies

$$
\left\langle A_{\omega}(\mathbf{x}), z\right\rangle=\left\langle\sum_{i=1}^{r} \omega_{i} x_{i}, z\right\rangle=\sum_{i=1}^{r} \omega_{i}\left\langle x_{i}, z\right\rangle .
$$

Let $M_{i}$ be a nontrivial $\left(M_{i} \neq\{0\}\right)$ closed and linear subspace of $\mathcal{H}, i=1, \ldots, r$, and let

$$
M:=\bigcap_{i=1}^{r} M_{i} .
$$

For each $\omega \in \Omega_{r}$, the simultaneous projection operator $T_{\omega}: \mathcal{H} \rightarrow \mathcal{H}$ is defined by

$$
T_{\omega}(x):=\sum_{i=1}^{r} \omega_{i} P_{M_{i}}(x),
$$

where $x \in \mathcal{H}$. Note that $T_{\omega}(x)=A_{\omega}\left(\left\{P_{M_{i}}(x)\right\}_{i=1}^{r}\right)$ and $\sum_{i=1}^{r} \omega_{i}\left\|P_{M_{i}}(x)\right\|^{2} \leq \sum_{i=1}^{r} \omega_{i}\|x\|^{2}=\|x\|^{2}<\infty$ due to the equalities $\left\|P_{M_{i}}\right\|=1, i=1, \ldots, r$; see, for example, [17, Theorem 5.13]. Hence, for $r=\infty$, the series $T_{\omega}(x)$ is absolutely convergent by Proposition 2.2. Moreover, since each projection $P_{M_{i}}$ is selfadjoint, see again [17, Theorem 5.13], this also holds for the simultaneous projection $T_{\omega}$. Furthermore, by the convexity of $\|\cdot\|$, we have $\left\|T_{\omega}\right\| \leq 1$ and Fix $T_{\omega}=M$. Indeed, the inclusion $M \subset \operatorname{Fix} T_{\omega}$ is obvious and if there is $x \in \operatorname{Fix} T_{\omega}$ such that $x \notin M_{j}$ for some $j \in\{1, \ldots, r\}$, then $\left\|P_{M_{j}}(x)\right\|<\|x\|$ and thus we arrive at a contradiction as $\|x\|=\left\|T_{\omega}(x)\right\| \leq \sum_{i=1}^{r} \omega_{i}\left\|P_{M_{i}}(x)\right\|<\|x\|$.

Following (1.3), for each $\omega \in \Omega_{r}$, we define the cosine of the Friedrichs angle between two nontrivial closed and linear subspaces $\mathbf{M}_{1}, \mathbf{M}_{2}$ of $\mathbf{H}_{\omega}$ by

$$
\cos _{\omega}\left(\mathbf{M}_{1}, \mathbf{M}_{2}\right):=\sup \left\{\langle\mathbf{x}, \mathbf{y}\rangle_{\omega}: \begin{array}{l}
\mathbf{x} \in \mathbf{M}_{1} \cap\left(\mathbf{M}_{1} \cap \mathbf{M}_{2}\right)^{\perp_{\omega}} \cap \mathbf{B}_{\omega}, \\
\mathbf{y} \in \mathbf{M}_{2} \cap\left(\mathbf{M}_{1} \cap \mathbf{M}_{2}\right)^{\perp_{\omega}} \cap \mathbf{B}_{\omega}
\end{array}\right\} \in[0,1],
$$

where $\mathbf{B}_{\omega}:=\left\{\mathbf{x} \in \mathbf{H}_{\omega}:\|\mathbf{x}\|_{\omega} \leq 1\right\}$. We use the symbol " $\perp_{\omega}$ " for the orthogonal complement in $\mathbf{H}_{\omega}$ which, for a closed linear subspace $\mathbf{M}$ of $\mathbf{H}_{\omega}$, is defined by

$$
\mathbf{M}^{\perp_{\omega}}:=\left\{\mathbf{x} \in \mathbf{H}_{\omega}:\langle\mathbf{x}, \mathbf{y}\rangle_{\omega}=0 \text { for all } \mathbf{y} \in \mathbf{M}\right\}
$$

see also Remark 2.3.

We now extend the definition for the product set $\mathbf{C}_{r}$ and the diagonal set $\mathbf{D}_{r}$ to

$$
\mathbf{C}_{\omega}:= \begin{cases}\prod_{i=1}^{r} M_{i}, & \text { if } r \in \mathbb{Z}_{+} \\ \left\{\left\{x_{i}\right\}_{i=1}^{\infty}: x_{i} \in M_{i}, i=1,2, \ldots, \sum_{i=1}^{\infty} \omega_{i}\left\|x_{i}\right\|^{2}<\infty\right\}, & \text { if } r=\infty .\end{cases}
$$

and

$$
\mathbf{D}_{\omega}:=\left\{\{x\}_{i=1}^{r}: x \in \mathcal{H}\right\}
$$

respectively, where $\omega \in \Omega_{r}$. It is not difficult to see that both $\mathbf{C}_{\omega}$ and $\mathbf{D}_{\omega}$ are closed and linear subspaces of $\mathbf{H}_{\omega}$. In this paper we are interested in the cosine between $\mathbf{C}_{\omega}$ and $\mathbf{D}_{\omega}$ and its connection to

$$
\mathbf{M}_{\omega}:= \begin{cases}\prod_{i=1}^{r} M, & \text { if } r \in \mathbb{Z}_{+} \\ \left\{\left\{x_{i}\right\}_{i=1}^{\infty}: x_{i} \in M, i=1,2, \ldots, \sum_{i=1}^{\infty} \omega_{i}\left\|x_{i}\right\|^{2}<\infty\right\}, & \text { if } r=\infty\end{cases}
$$


and

$$
\boldsymbol{\Delta}_{\omega}:=\prod_{i=1}^{r} B
$$

where $B:=\{x \in \mathcal{H}:\|x\| \leq 1\}$. For this reason, we introduce the following configuration constant:

$$
c_{\omega}\left(\mathbf{M}_{1}, \mathbf{M}_{2}\right):=\sup \left\{\langle\mathbf{x}, \mathbf{y}\rangle_{\omega}: \begin{array}{l}
\mathbf{x} \in \mathbf{M}_{1} \cap\left(\mathbf{M}_{1} \cap \mathbf{M}_{2}\right)^{\perp_{\omega}} \cap \boldsymbol{\Delta}_{\omega} \\
\mathbf{y} \in \mathbf{M}_{2} \cap\left(\mathbf{M}_{1} \cap \mathbf{M}_{2}\right)^{\perp_{\omega}} \cap \boldsymbol{\Delta}_{\omega}
\end{array}\right\} \in[0,1]
$$

Remark 2.3 (Space in Question for " $\perp$ "). In our further study, by fixing $\omega \in \Omega_{r}$, we restrict our analysis only to the Hilbert space $\left(\mathbf{H}_{\omega},\langle\cdot, \cdot\rangle_{\omega}\right)$. In particular, when $r=\infty$, we consider the space $\left(\ell_{\omega}^{2}(\mathcal{H}),\langle\cdot, \cdot\rangle_{\omega}\right)$. Therefore, by using the subscript " $\omega$ " added to a set, we implicitly assume that such a set is considered in $\left(\mathbf{H}_{\omega},\langle\cdot, \cdot\rangle_{\omega}\right)$. Knowing the underlying space becomes very important especially for the operation of the orthogonal complement. For example, the diagonal set $\mathbf{D}_{\omega}$ is the same for all $\omega \in \Omega_{r}$ while its orthogonal complement $\left(\mathbf{D}_{\omega}\right)^{\perp_{\omega}}$ may be different for different $\omega$ 's (see Proposition 2.5). Having this in mind, we may simply write " $\perp$ " instead of " $\perp_{\omega}$ " when the underlying space $\mathbf{H}_{\omega}$ is known from the context. For example, $\mathbf{D}_{\omega}^{\perp}$ reads as $\left(\mathbf{D}_{\omega}\right)^{\perp_{\omega}}$.

We proceed with the following proposition.

Proposition 2.4. Let $\omega \in \Omega_{r}$. We have

$$
\mathbf{M}_{\omega}^{\perp}= \begin{cases}\prod_{i=1}^{r} M^{\perp}, & \text { if } r \in \mathbb{Z}_{+} \\ \left\{\left\{x_{i}\right\}_{i=1}^{\infty}: x_{i} \in M^{\perp}, i=1,2, \ldots, \sum_{i=1}^{\infty} \omega_{i}\left\|x_{i}\right\|^{2}<\infty\right\}, & \text { if } r=\infty .\end{cases}
$$

and $\mathbf{D}_{\omega} \cap \mathbf{M}_{\omega}^{\perp}=\mathbf{D}_{\omega} \cap\left(\mathbf{C}_{\omega} \cap \mathbf{D}_{\omega}\right)^{\perp}$. However, the inclusion

$$
\mathbf{C}_{\omega} \cap \mathbf{M}_{\omega}^{\perp} \subset \mathbf{C}_{\omega} \cap\left(\mathbf{C}_{\omega} \cap \mathbf{D}_{\omega}\right)^{\perp}
$$

is strict if $M \neq\{0\}$ and $M_{j} \neq M$ for some $j \in\{1, \ldots, r\}$.

Proof. We begin by showing the first equality. We denote the set on the right-hand side of (2.16) by $\mathbf{M}_{\omega}^{\prime}$. It is not difficult to see that $\mathbf{M}_{\omega}^{\perp} \supset \mathbf{M}_{\omega}^{\prime}$. We now demonstrate the opposite inclusion " $\subset$ ". Indeed, let $\mathbf{x}=\left\{x_{i}\right\}_{i=1}^{r} \in \mathbf{M}_{\omega}^{\perp}$. Then, by (2.10), $\langle\mathbf{x}, \mathbf{y}\rangle_{\omega}=0$ for all $\mathbf{y}=\left\{y_{i}\right\}_{i=1}^{r} \in \mathbf{M}_{\omega}$, where $y_{i} \in M$. By choosing $j \in\{1, \ldots, r\}$ and $y_{i}:=0$ for all $i \neq j$, we obtain $\left\langle x_{j}, y_{j}\right\rangle=0$ for all $y_{j} \in M$, and thus we must have $x_{j} \in M^{\perp}$. The arbitrariness of $j$ implies that $x_{j} \in M^{\perp}$ for all $j \in\{1, \ldots, r\}$. Note that up to now, the above-presented argument holds for $r \in \mathbb{Z}_{+}$as well as for $r=\infty$. In the latter case $(r=\infty)$, by (2.10), we see that, $\mathbf{x} \in \mathbf{H}_{\omega}$. Consequently, $\sum_{i=1}^{\infty} \omega_{i}\left\|x_{i}\right\|^{2}<\infty$. This shows that $\mathbf{x} \in \mathbf{M}_{\omega}^{\prime}$, as asserted.

Next we show the second equality and inclusion (2.17). To this end, observe that, by the definition of $\mathbf{C}_{\omega}$ and $\mathbf{D}_{\omega}$, we get

$$
\mathbf{C}_{\omega} \cap \mathbf{D}_{\omega}=\left\{\{x\}_{i=1}^{r}: x \in M\right\} .
$$

Consequently, by using the first equality, we see that

$$
\begin{aligned}
\mathbf{D}_{\omega} \cap \mathbf{M}_{\omega}^{\perp} & =\left\{\{x\}_{i=1}^{r}: x \in M^{\perp}\right\} \\
& =\left\{\{x\}_{i=1}^{r}:\langle x, y\rangle=0 \text { for all } y \in M\right\} \\
& =\left\{\mathbf{x} \in \mathbf{D}_{\omega}:\langle\mathbf{x}, \mathbf{y}\rangle_{\omega}=0 \text { for all } \mathbf{y} \in \mathbf{C}_{\omega} \cap \mathbf{D}_{\omega}\right\} \\
& =\mathbf{D}_{\omega} \cap\left(\mathbf{C}_{\omega} \cap \mathbf{D}_{\omega}\right)^{\perp}
\end{aligned}
$$


and

$$
\begin{aligned}
\mathbf{C}_{\omega} \cap \mathbf{M}_{\omega}^{\perp} & =\left\{\left\{x_{i}\right\}_{i=1}^{r} \in \mathbf{H}_{\omega}: x_{i} \in M_{i} \cap M^{\perp}, i=1, \ldots, r\right\} \\
& \subset\left\{\left\{x_{i}\right\}_{i=1}^{r} \in \mathbf{H}_{\omega}: x_{i} \in M_{i}, i=1, \ldots, r, \sum_{i=1}^{r} \omega_{i} x_{i} \in M^{\perp}\right\} \\
& =\mathbf{C}_{\omega} \cap\left(\mathbf{C}_{\omega} \cap \mathbf{D}_{\omega}\right)^{\perp} .
\end{aligned}
$$

Finally, we show that inclusion (2.17) is strict. The assumption $M_{j} \neq M$ guarantees that the subspace $M_{j} \cap M^{\perp}$ is nontrivial. Indeed, by the orthogonal decomposition theorem (that is, $I=P_{M}+P_{M^{\perp}}$ ), for any $x \in M_{j} \backslash M$, the point $x_{j}:=P_{M^{\perp}}(x)=x-P_{M}(x) \neq 0$ and $x_{j} \in M_{j} \cap M^{\perp}$.

Let $m \in M$ be nonzero. Define $\mathbf{y}=\left\{y_{i}\right\}_{i=1}^{r}$ by $y_{j}:=\frac{1}{\omega_{j}}\left(m+x_{j}\right), y_{j+1}:=-\frac{1}{\omega_{j+1}} m$ and $y_{i}:=0$ for all $i \neq j, j+1$. Note that $y_{j} \notin M^{\perp}$. Thus $\mathbf{y} \notin \mathbf{C}_{\omega} \cap \mathbf{M}_{\omega}^{\perp}$. On the other hand, $y_{i} \in M_{i}$ for all $i=1, \ldots, r$, and, moreover, for any $m^{\prime} \in M$, we have

$$
\sum_{i=1}^{r} \omega_{i}\left\langle y_{i}, m^{\prime}\right\rangle=\left\langle\left(m+x_{j}\right)-m, m^{\prime}\right\rangle=\left\langle x_{j}, m^{\prime}\right\rangle=0
$$

which proves that $\mathbf{y} \in \mathbf{C}_{\omega} \cap\left(\mathbf{C}_{\omega} \cap \mathbf{D}_{\omega}\right)^{\perp}$.

In the next proposition we show the connections among the sets $\mathbf{C}_{\omega}, \mathbf{D}_{\omega}$ and the averaging operator $A_{\omega}$. When $r \in \mathbb{Z}_{+}$, equality (2.24) can be found in [17, Theorem $\left.4.6(5)\right]$.

Proposition 2.5. Let $\omega \in \Omega_{r}$. We have

$$
\mathbf{C}_{\omega}^{\perp}= \begin{cases}\prod_{i=1}^{r} M_{i}^{\perp}, & \text { if } r \in \mathbb{Z}_{+} \\ \left\{\left\{x_{i}\right\}_{i=1}^{\infty}: x_{i} \in M_{i}^{\perp}, i=1,2, \ldots, \sum_{i=1}^{\infty} \omega_{i}\left\|x_{i}\right\|^{2}<\infty\right\}, & \text { if } r=\infty\end{cases}
$$

and $\mathbf{D}_{\omega}^{\perp}=\mathcal{N}\left(A_{\omega}\right)$ - the null space of $A_{\omega}$. Consequently, the subspace $\mathbf{C}_{\omega}^{\perp}+\mathbf{D}_{\omega}^{\perp}$ is closed in $\mathbf{H}_{\omega}$ if and only if the subspace

$$
A_{\omega}\left(\mathbf{C}_{\omega}^{\perp}\right)= \begin{cases}\sum_{i=1}^{r} M_{i}^{\perp}, & \text { if } r \in \mathbb{Z}_{+}, \\ \left\{\sum_{i=1}^{\infty} \omega_{i} x_{i}: x_{i} \in M_{i}^{\perp}, i=1,2, \ldots, \sum_{i=1}^{\infty} \omega_{i}\left\|x_{i}\right\|^{2}<\infty\right\}, & \text { if } r=\infty\end{cases}
$$

is closed in $\mathcal{H}$. Moreover,

$$
\overline{A_{\omega}\left(\mathbf{C}_{\omega}^{\perp}\right)}=M^{\perp} .
$$

Proof. The first equality can be established by using an argument similar to the one presented in the proof of Proposition 2.4 with $M$ replaced by $M_{j}$.

In order to show the second equality, take $\mathbf{x} \in \mathbf{D}_{\omega}^{\perp}$. Then, by (2.6), for all $\mathbf{y}=\{y\}_{i=1}^{r} \in \mathbf{D}_{\omega}$, we have $\langle\mathbf{x}, \mathbf{y}\rangle_{\omega}=\left\langle A_{\omega}(\mathbf{x}), y\right\rangle=0$. In particular, by taking $y:=A_{\omega}(\mathbf{x})$, we see that $A_{\omega}(\mathbf{x})=0$, that is, $\mathbf{x} \in \mathcal{N}\left(A_{\omega}\right)$. On the other hand, it is easy to see that when $\mathbf{x} \in \mathcal{N}\left(A_{\omega}\right)$, then for all $\mathbf{y}=\{y\}_{i=1}^{r} \in \mathbf{D}_{\omega}$, we have $0=\left\langle A_{\omega}(\mathbf{x}), y\right\rangle=\langle\mathbf{x}, \mathbf{y}\rangle_{\omega}$, that is, $\mathbf{x} \in \mathbf{D}_{\omega}^{\perp}$. This shows the second equality.

Recall that $A_{\omega}$ is linear and bounded. In view of $\left[23\right.$, section $17 \mathrm{H}$, p. 142], the set $A_{\omega}\left(\mathbf{C}_{\omega}^{\perp}\right)$ is closed in $\mathcal{H}$ if and only if $\mathbf{C}_{\omega}^{\perp}+\mathcal{N}\left(A_{\omega}\right)$ is closed in $\mathbf{H}_{\omega}$. The equalities in (2.23) follow from the above discussion.

We now focus on (2.24), where we first show that

$$
\left(A_{\omega}\left(\mathbf{C}_{\omega}^{\perp}\right)\right)^{\perp}=M .
$$


It is not difficult to see that $M \subset\left(A_{\omega}\left(\mathbf{C}_{\omega}^{\perp}\right)\right)^{\perp}$. In order to demonstrate the opposite inclusion " $\supset$ ", take $x \in\left(A_{\omega}\left(\mathbf{C}_{\omega}^{\perp}\right)\right)^{\perp}$. Consequently, for all $\mathbf{y}=\left\{y_{i}\right\}_{i=1}^{r} \in \mathbf{C}_{\omega}^{\perp}$ (hence $y_{i} \in M_{i}^{\perp}$ ), we have $\left\langle x, A_{\omega}(\mathbf{y})\right\rangle=0$. In particular, by choosing $j \in\{1, \ldots, r\}$ and setting $y_{i}:=0$ for all $i \neq j$, we obtain that $\left\langle x, y_{j}\right\rangle=0$ for all $y_{j} \in M_{j}^{\perp}$. This, when combined with the fact that $M_{j}$ is a closed linear subspace, implies that $x \in M_{j}^{\perp \perp}=M_{j}$; see, for example, [17, Theorem 4.5 (8)]. The arbitrariness of $j \in\{1, \ldots, r\}$ yields that $x \in M$.

We now return to (2.24). Recall that, when $L$ is a linear subspace of $\mathcal{H}$ which is not necessarily closed, then $L^{\perp}=(\bar{L})^{\perp}$; see $[17$, Theorem $4.5(2)]$. Consequently, by $(2.25)$,

$$
M^{\perp}=\left(A_{\omega}\left(\mathbf{C}_{\omega}^{\perp}\right)\right)^{\perp \perp}=\left(\overline{A_{\omega}\left(\mathbf{C}_{\omega}^{\perp}\right)}\right)^{\perp \perp}=\overline{A_{\omega}\left(\mathbf{C}_{\omega}^{\perp}\right)}
$$

which completes the proof.

Note that similarly to Example 2.1, when $r=\infty$, the sets $A_{\omega}\left(\mathbf{C}_{\omega}^{\perp}\right)$ may be different for different $\omega \in \Omega_{\infty}$.

Example 2.6 $(r=\infty)$. Assume that $\mathcal{H}$ is separable and let $\left\{e_{i}\right\}_{i=1}^{\infty}$ be a norm-one Schauder basis of it. Let $M_{i}^{\perp}:=\operatorname{span}\left\{e_{i}\right\}$. Using the notation of Example 2.1, consider two spaces, $\mathbf{H}_{\omega, \beta}$ and $\mathbf{H}_{\omega, \beta^{\prime}}$, with

$$
\varepsilon>0, \quad \alpha>0, \quad \beta:=1+\alpha+\varepsilon \quad \text { and } \quad \alpha^{\prime}:=\alpha+2 \varepsilon, \quad \beta^{\prime}:=1+\alpha^{\prime} .
$$

By using Example 2.1, we see that

$$
\mathbf{x}_{\alpha}:=\left\{i^{\alpha / 2} e_{i}\right\}_{i=1}^{\infty} \in \mathbf{H}_{\omega, \beta} \quad \text { and } \quad \mathbf{x}_{\alpha^{\prime}}:=\left\{i^{\alpha^{\prime} / 2} e_{i}\right\}_{i=1}^{\infty} \notin \mathbf{H}_{\omega, \beta^{\prime}}
$$

Consequently, $y:=\frac{s_{\beta}}{s_{\beta^{\prime}}} A_{\omega, \beta}\left(\mathbf{x}_{\alpha}\right) \in A_{\omega, \beta}\left(\mathbf{C}_{\omega, \beta}^{\perp}\right)$. Note that, by the choice of the $e_{i}$ 's, the representation of

$$
y=\frac{1}{s_{\beta^{\prime}}} \sum_{i=1}^{\infty} \frac{1}{i^{\beta^{\prime}}}\left(i^{\left[\alpha+2\left(\beta^{\prime}-\beta\right)\right] / 2} e_{i}\right)=\frac{1}{s_{\beta^{\prime}}} \sum_{i=1}^{\infty} \frac{1}{i^{\beta^{\prime}}}\left(i^{\alpha^{\prime} / 2} e_{i}\right)
$$

is unique. Hence, by (2.28), we see that $y \notin A_{\omega, \beta^{\prime}}\left(\mathbf{C}_{\omega, \beta^{\prime}}^{\perp}\right)$.

Remark 2.7 (Notation). To emphasize that we refer to a particular vector $\omega=\{1 / r, \ldots, 1 / r\} \in \Omega_{r}$ for some $r \in \mathbb{Z}_{+}$, we may replace the subscript " $\omega$ " by the subscript " $r$ " in all the above-mentioned definitions. For example, we write

$$
\mathbf{H}_{r},\langle\cdot, \cdot\rangle_{r},\|\cdot\|_{r}, \quad \mathbf{C}_{r}, \mathbf{D}_{r}, \mathbf{M}_{r}, \boldsymbol{\Delta}_{r}, \quad \cos _{r}(\cdot, \cdot), c_{r}(\cdot, \cdot), \quad T_{r} \text { and } A_{r}
$$

instead of

$$
\mathbf{H}_{\omega},\langle\cdot, \cdot\rangle_{\omega},\|\cdot\|_{\omega}, \quad \mathbf{C}_{\omega}, \mathbf{D}_{\omega}, \mathbf{M}_{\omega}, \boldsymbol{\Delta}_{\omega}, \quad \cos _{\omega}(\cdot, \cdot), c_{\omega}(\cdot, \cdot), \quad T_{\omega} \text { and } A_{\omega},
$$

respectively. This coincides with the notation used in the introduction.

\section{Alternating Projection Formalization of Pierra}

In the next two results we bring out the connections among the operators $A_{\omega}, P_{M}$ and $T_{\omega}$, and the projections $P_{\mathbf{C}_{\omega}}, P_{\mathbf{D}_{\omega}}$ and $P_{\mathbf{C}_{\omega} \cap \mathbf{D}_{\omega}}$. 
Lemma 3.1. Let $\omega \in \Omega_{r}$. For each $\mathbf{x}:=\left\{x_{i}\right\}_{i=1}^{r} \in \mathbf{H}_{\omega}$, we have

$$
\begin{gathered}
P_{\mathbf{C}_{\omega}}(\mathbf{x})=\left\{P_{M_{i}}\left(x_{i}\right)\right\}_{i=1}^{r}, \\
P_{\mathbf{D}_{\omega}}(\mathbf{x})=\left\{A_{\omega}(\mathbf{x})\right\}_{i=1}^{r}
\end{gathered}
$$

and

$$
P_{\mathbf{C}_{\omega} \cap \mathbf{D}_{\omega}}(\mathbf{x})=\left\{P_{M}\left(A_{\omega}(\mathbf{x})\right)\right\}_{i=1}^{r} .
$$

Proof. Recall that for a closed and linear subspace $L$ of $\mathcal{H}$, we have

$$
y=P_{L}(x) \Longleftrightarrow y \in L \quad \text { and } \quad\langle x-y, z\rangle=0 \quad \forall z \in L
$$

see, for example, [17, Theorem 4.9]. Analogously, for a closed and linear subspace $\mathbf{L}$ of $\mathbf{H}_{\omega}$, we have

$$
\mathbf{y}=P_{\mathbf{L}}(\mathbf{x}) \quad \Longleftrightarrow \quad \mathbf{y} \in \mathbf{L} \quad \text { and } \quad\langle\mathbf{x}-\mathbf{y}, \mathbf{z}\rangle_{\omega}=0 \quad \forall \mathbf{z} \in \mathbf{L}
$$

We now consider each asserted equality separately.

By definition, the point $\mathbf{y}:=\left\{P_{M_{i}}\left(x_{i}\right)\right\}_{i=1}^{r}$ satisfies $P_{M_{i}}\left(x_{i}\right) \in M_{i}, i=1, \ldots, r$. Moreover, using the equality $\left\|P_{M_{i}}\right\|=1$, we see that

$$
\sum_{i=1}^{r} \omega_{i}\left\|P_{M_{i}}\left(x_{i}\right)\right\|^{2} \leq \sum_{i=1}^{r} \omega_{i}\left\|x_{i}\right\|^{2}=\|\mathbf{x}\|_{\omega}^{2}<\infty .
$$

This shows that $\mathbf{y} \in \mathbf{C}_{\omega}$. Furthermore, by (3.4) applied to $L:=M_{i}, i=1, \ldots, r$, for each $\mathbf{z}=\left\{z_{i}\right\}_{i=1}^{r} \in$ $\mathbf{C}_{\omega}$, we have

$$
\langle\mathbf{x}-\mathbf{y}, \mathbf{z}\rangle_{\omega}=\sum_{i=1}^{r} \omega_{i}\left\langle x_{i}-P_{M_{i}}\left(x_{i}\right), z_{i}\right\rangle=0 .
$$

By (3.5), this shows (3.1).

By definition, $\mathbf{y}:=\left\{A_{\omega}(\mathbf{x})\right\}_{i=1}^{r} \in \mathbf{D}_{\omega}$. Moreover, by (2.6), for each $\mathbf{z}=\{z\}_{i=1}^{r} \in \mathbf{D}_{\omega}$, we have

$$
\langle\mathbf{x}-\mathbf{y}, \mathbf{z}\rangle_{\omega}=\sum_{i=1}^{r} \omega_{i}\left\langle x_{i}-A_{\omega}(\mathbf{x}), z\right\rangle=\left\langle\sum_{i=1}^{r} \omega_{i}\left(x_{i}-A_{\omega}(\mathbf{x})\right), z\right\rangle=\left\langle A_{\omega}(\mathbf{x})-A_{\omega}(\mathbf{x}), z\right\rangle=0 .
$$

Again, by (3.5), this proves (3.2).

Finally, let now $\mathbf{y}:=\left\{P_{M}\left(A_{\omega}(\mathbf{x})\right)\right\}_{i=1}^{r}$. It is clear that, by definition, $\mathbf{y} \in \mathbf{C}_{\omega} \cap \mathbf{D}_{\omega}=\left\{\mathbf{x}=\{x\}_{i=1}^{r}: x \in\right.$ $M\}$. By (2.6) and (3.4), for any $\mathbf{z}=\{z\}_{i=1}^{r} \in \mathbf{C}_{\omega} \cap \mathbf{D}_{\omega}$, we have

$$
\begin{aligned}
\langle\mathbf{x}-\mathbf{y}, \mathbf{z}\rangle_{\omega} & =\sum_{i=1}^{r} \omega_{i}\left\langle x_{i}-P_{M}\left(A_{\omega}(\mathbf{x})\right), z\right\rangle=\left\langle\sum_{i=1}^{r} \omega_{i}\left(x_{i}-P_{M}\left(A_{\omega}(\mathbf{x})\right)\right), z\right\rangle \\
& =\left\langle A_{\omega}(\mathbf{x})-P_{M}\left(A_{\omega}(\mathbf{x})\right), z\right\rangle=0 .
\end{aligned}
$$

This, in view of (3.5), proves the last equality.

Theorem 3.2. Let $\omega \in \Omega_{r}$. For each $x \in \mathcal{H}$ and $\mathbf{x}:=\{x\}_{i=1}^{r}$, we have

$$
\left\|T_{\omega}^{k}(x)-P_{M}(x)\right\|=\left\|\left(P_{\mathbf{D}_{\omega}} P_{\mathbf{C}_{\omega}}\right)^{k}(\mathbf{x})-P_{\mathbf{C}_{\omega} \cap \mathbf{D}_{\omega}}(\mathbf{x})\right\|_{\omega} \rightarrow 0 \quad \text { as } k \rightarrow \infty .
$$


Proof. Using (3.1), (3.2) and induction with respect to $k$, we see that the equality

$$
\left(P_{\mathbf{D}_{\omega}} P_{\mathbf{C}_{\omega}}\right)^{k}(\mathbf{x})=\left\{T_{\omega}^{k}(x)\right\}_{i=1}^{r}
$$

holds for all $x \in \mathcal{H}$ and $\mathbf{x}:=\{x\}_{i=1}^{r} \in \mathbf{H}_{\omega}$. This, when combined with (3.3), leads to

$$
\left\|T_{\omega}^{k}(x)-P_{M}(x)\right\|=\left\|\left\{T_{\omega}^{k}(x)\right\}_{i=1}^{r}-\left\{P_{M}(x)\right\}_{i=1}^{r}\right\|_{\omega}=\left\|\left(P_{\mathbf{D}_{\omega}} P_{\mathbf{C}_{\omega}}\right)^{k}(\mathbf{x})-P_{\mathbf{C}_{\omega} \cap \mathbf{D}_{\omega}}(\mathbf{x})\right\|_{\omega},
$$

which proves the equality in (3.10). The norm convergence of the alternating projection method follows from Theorem 1.1 applied to $M_{1}:=\mathbf{C}_{\omega}$ and $M_{2}:=\mathbf{D}_{\omega}$ in $\mathbf{H}_{\omega}$.

Theorem 3.3 (Exact Norm Value). Let $\omega \in \Omega_{r}$. For each $k=1,2, \ldots$, we have

$$
\left\|T_{\omega}^{k}-P_{M}\right\|=\left\|\left(P_{\mathbf{D}_{\omega}} P_{\mathbf{C}_{\omega}} P_{\mathbf{D}_{\omega}}\right)^{k}-P_{\mathbf{C}_{\omega} \cap \mathbf{D}_{\omega}}\right\|_{\omega}=\cos _{\omega}\left(\mathbf{C}_{\omega}, \mathbf{D}_{\omega}\right)^{2 k} \leq 1 .
$$

Proof. The proof follows the argument in [33, Theorem 7]. We give it here for the convenience of the reader.

Recall that the operator $T_{\omega}$ is self-adjoint, $\left\|T_{\omega}\right\| \leq 1$ and Fix $T_{\omega}=M$ (compare with Section 2). Moreover, for each $i=1, \ldots, r$, the projection $P_{M_{i}}$ commutes with $P_{M}$, that is,

$$
P_{M} P_{M_{i}}=P_{M_{i}} P_{M}=P_{M}
$$

see [17, Lemma 9.2]. Consequently, the operator $T_{\omega}$ commutes with $P_{M}$ too and we have

$$
P_{M} T_{\omega}=T_{\omega} P_{M}=P_{M}
$$

By using [33, Lemma 6], we get $\left\|T_{\omega}^{k}-P_{M}\right\|=\left\|T_{\omega}-P_{M}\right\|^{k}$.

On the other hand, the operator $\mathbf{T}:=P_{\mathbf{D}_{\omega}} P_{\mathbf{C}_{\omega}} P_{\mathbf{D}_{\omega}}$ is also self-adjoint, $\|\mathbf{T}\|_{\omega} \leq 1$ and Fix $\mathbf{T}=$ $\mathbf{C}_{\omega} \cap \mathbf{D}_{\omega}$. Note that similarly to (3.14), the projections $P_{\mathbf{C}_{\omega}}$ and $P_{\mathbf{D}_{\omega}}$ commute with $P_{\mathbf{C}_{\omega} \cap \mathbf{D}_{\omega}}$, where

$$
P_{\mathbf{C}_{\omega} \cap \mathbf{D}_{\omega}}=P_{\mathbf{C}_{\omega} \cap \mathbf{D}_{\omega}} P_{\mathbf{C}_{\omega}}=P_{\mathbf{C}_{\omega}} P_{\mathbf{C}_{\omega} \cap \mathbf{D}_{\omega}}=P_{\mathbf{C}_{\omega} \cap \mathbf{D}_{\omega}} P_{\mathbf{D}_{\omega}}=P_{\mathbf{D}_{\omega}} P_{\mathbf{C}_{\omega} \cap \mathbf{D}_{\omega}} .
$$

This leads to

$$
P_{\mathbf{C}_{\omega} \cap \mathbf{D}_{\omega}} \mathbf{T}=\mathbf{T} P_{\mathbf{C}_{\omega} \cap \mathbf{D}_{\omega}}=P_{\mathbf{C}_{\omega} \cap \mathbf{D}_{\omega}} .
$$

Again, by using [33, Lemma 6], but this time in $\mathbf{H}_{\omega}$, we obtain $\left\|\mathbf{T}^{k}-P_{\mathbf{C}_{\omega} \cap \mathbf{D}_{\omega}}\right\|_{\omega}=\left\|\mathbf{T}-P_{\mathbf{C}_{\omega} \cap \mathbf{D}_{\omega}}\right\|_{\omega}^{k}$.

In order to complete the proof, it suffices to show the equalities of (3.13) only for $k=1$. By the properties of the adjoint operation " $*$ " and by Theorem 1.5, we obtain

$$
\begin{aligned}
\left\|P_{\mathbf{D}_{\omega}} P_{\mathbf{C}_{\omega}} P_{\mathbf{D}_{\omega}}-P_{\mathbf{C}_{\omega} \cap \mathbf{D}_{\omega}}\right\|_{\omega} & =\left\|P_{\mathbf{D}_{\omega}} P_{\mathbf{C}_{\omega}} P_{\mathbf{C}_{\omega}} P_{\mathbf{D}_{\omega}}-P_{\mathbf{C}_{\omega} \cap \mathbf{D}_{\omega}}\right\|_{\omega} \\
& =\left\|\left(P_{\mathbf{D}_{\omega}} P_{\mathbf{C}_{\omega}}-P_{\mathbf{C}_{\omega} \cap \mathbf{D}_{\omega}}\right)\left(P_{\mathbf{C}_{\omega}} P_{\mathbf{D}_{\omega}}-P_{\mathbf{C}_{\omega} \cap \mathbf{D}_{\omega}}\right)\right\|_{\omega} \\
& =\left\|\left(P_{\mathbf{D}_{\omega}} P_{\mathbf{C}_{\omega}}-P_{\mathbf{C}_{\omega} \cap \mathbf{D}_{\omega}}\right)\left(P_{\mathbf{D}_{\omega}} P_{\mathbf{C}_{\omega}}-P_{\mathbf{C}_{\omega} \cap \mathbf{D}_{\omega}}\right)^{*}\right\|_{\omega} \\
& =\left\|P_{\mathbf{D}_{\omega}} P_{\mathbf{C}_{\omega}}-P_{\mathbf{C}_{\omega} \cap \mathbf{D}_{\omega}}\right\|_{\omega}^{2} \\
& =\cos _{\omega}\left(\mathbf{C}_{\omega}, \mathbf{D}_{\omega}\right)^{2} .
\end{aligned}
$$


Let $\mathbf{B}_{\omega}:=\left\{\mathbf{x}:\|\mathbf{x}\|_{\omega} \leq 1\right\}$. Since $P_{\mathbf{D}_{\omega}}\left(\mathbf{B}_{\omega}\right)=\mathbf{D}_{\omega} \cap \mathbf{B}_{\omega}$, we see that

$$
\begin{aligned}
\left\|P_{\mathbf{D}_{\omega}} P_{\mathbf{C}_{\omega}} P_{\mathbf{D}_{\omega}}-P_{\mathbf{C}_{\omega} \cap \mathbf{D}_{\omega}}\right\|_{\omega} & =\left\|P_{\mathbf{D}_{\omega}} P_{\mathbf{C}_{\omega}} P_{\mathbf{D}_{\omega}}-P_{\mathbf{C}_{\omega} \cap \mathbf{D}_{\omega}} P_{\mathbf{D}_{\omega}}\right\|_{\omega} \\
& =\sup \left\{\left\|P_{\mathbf{D}_{\omega}} P_{\mathbf{C}_{\omega}} P_{\mathbf{D}_{\omega}}(\mathbf{x})-P_{\mathbf{C}_{\omega} \cap \mathbf{D}_{\omega}} P_{\mathbf{D}_{\omega}}(\mathbf{x})\right\|_{\omega}: \mathbf{x} \in \mathbf{B}_{\omega}\right\} \\
& =\sup \left\{\left\|P_{\mathbf{D}_{\omega}} P_{\mathbf{C}_{\omega}}(\mathbf{y})-P_{\mathbf{C}_{\omega} \cap \mathbf{D}_{\omega}}(\mathbf{y})\right\|_{\omega}: \mathbf{y} \in P_{\mathbf{D}_{\omega}}\left(\mathbf{B}_{\omega}\right)\right\} \\
& =\sup \left\{\left\|P_{\mathbf{D}_{\omega}} P_{\mathbf{C}_{\omega}}(\mathbf{y})-P_{\mathbf{C}_{\omega} \cap \mathbf{D}_{\omega}}(\mathbf{y})\right\|_{\omega}: \mathbf{y} \in \mathbf{D}_{\omega} \cap \mathbf{B}_{\omega}\right\} \\
& =\sup \left\{\left\|T_{\omega}(y)-P_{M}(y)\right\|: y \in \mathcal{H} \text { and }\|y\| \leq 1\right\} \\
& =\left\|T_{\omega}-P_{M}\right\| .
\end{aligned}
$$

This completes the proof.

Note that equality (3.13) becomes useful only when $\cos _{\omega}\left(\mathbf{C}_{\omega}, \mathbf{D}_{\omega}\right)<1$ in which case it turns into the optimal error bound. We return to this inequality in Theorems 4.9 and 5.1 below.

\section{Properties of the Cosine $\cos _{\omega}\left(\mathbf{C}_{\omega}, \mathbf{D}_{\omega}\right)$}

In the next two lemmata, we show that the set $\left(\mathbf{C}_{\omega} \cap \mathbf{D}_{\omega}\right)^{\perp}$ can be replaced by its subset $\mathbf{M}_{\omega}^{\perp}$ in the definitions of $\cos _{\omega}\left(\mathbf{C}_{\omega}, \mathbf{D}_{\omega}\right)$ and $c_{\omega}\left(\mathbf{C}_{\omega}, \mathbf{D}_{\omega}\right)$, despite the discussion concerning the inclusion of Proposition 2.4 .

Lemma 4.1. Let $\omega \in \Omega_{r}$. The cosine of the Friedrichs angle between $\mathbf{C}_{\omega}$ and $\mathbf{D}_{\omega}$ satisfies:

$$
\begin{aligned}
& \cos _{\omega}\left(\mathbf{C}_{\omega}, \mathbf{D}_{\omega}\right)=\sup \left\{\langle\mathbf{x}, \mathbf{y}\rangle_{\omega}: \begin{array}{l}
\mathbf{x} \in \mathbf{C}_{\omega} \cap \mathbf{M}_{\omega}^{\perp} \cap \mathbf{B}_{\omega}, \\
\mathbf{y} \in \mathbf{D}_{\omega} \cap \mathbf{M}_{\omega}^{\perp} \cap \mathbf{B}_{\omega}
\end{array}\right\} \\
& =\sup \left\{\sum_{i=1}^{r} \omega_{i}\left\langle x_{i}, y\right\rangle: \begin{array}{l}
x_{i} \in M_{i} \cap M^{\perp}, i=1, \ldots, r, \sum_{i=1}^{r} \omega_{i}\left\|x_{i}\right\|^{2} \leq 1, \\
y \in M^{\perp},\|y\| \leq 1
\end{array}\right\} .
\end{aligned}
$$

Proof. Denote the right-hand side of (4.1) by $\alpha$ and observe that, by the inclusion $\mathbf{C}_{\omega} \cap \mathbf{M}_{\omega}^{\perp} \subset \mathbf{C}_{\omega} \cap$ $\left(\mathbf{C}_{\omega} \cap \mathbf{D}_{\omega}\right)^{\perp}$, we have $\cos _{\omega}\left(\mathbf{C}_{\omega}, \mathbf{D}_{\omega}\right) \geq \alpha$. We now show that $\cos _{\omega}\left(\mathbf{C}_{\omega}, \mathbf{D}_{\omega}\right) \leq \alpha$.

Note first that, analogously to (3.14), for each $i=1, \ldots, r$, we have

$$
P_{M \perp} P_{M_{i}}=P_{M_{i}} P_{M \perp}=P_{M_{i} \cap M^{\perp}} .
$$

Indeed, when $x \in M_{i}$, by (3.14) and using the orthogonal decomposition theorem, we see that

$$
P_{M^{\perp}}(x)=P_{M^{\perp}} P_{M_{i}}(x)=P_{M_{i}}(x)-P_{M} P_{M_{i}}(x)=P_{M_{i}}(x)-P_{M}(x) \in M_{i},
$$

that is, $P_{M^{\perp}}\left(M_{i}\right) \subset M_{i}$. Hence we may again apply [17, Lemma 9.2] to obtain (4.2).

Let $\mathbf{x}=\left\{x_{i}\right\}_{i=1}^{r} \in \mathbf{H}_{\omega}$ be such that $x_{i} \in M_{i}, i=1, \ldots, r$, and let $y \in M^{\perp}$. Using (4.2), we arrive at

$$
\left\langle x_{i}, y\right\rangle=\left\langle P_{M}\left(x_{i}\right)+P_{M^{\perp}}\left(x_{i}\right), y\right\rangle=\left\langle P_{M^{\perp}} P_{M_{i}}\left(x_{i}\right), y\right\rangle=\left\langle P_{M_{i} \cap M^{\perp}}\left(x_{i}\right), y\right\rangle .
$$

Furthermore, by (3.14) and (4.2), we obtain

$$
\left\|x_{i}\right\|^{2}=\left\|P_{M_{i} \cap M}\left(x_{i}\right)\right\|^{2}+\left\|P_{M_{i} \cap M^{\perp}}\left(x_{i}\right)\right\|^{2} .
$$


Therefore,

$$
\begin{aligned}
\cos _{\omega}\left(\mathbf{C}_{\omega}, \mathbf{D}_{\omega}\right) & =\sup \left\{\sum_{i=1}^{r} \omega_{i}\left\langle x_{i}, y\right\rangle: \begin{array}{l}
x_{i} \in M_{i}, i=1, \ldots, r, \sum_{i=1}^{r} \omega_{i} x_{i} \in M^{\perp}, \\
\sum_{i=1}^{r} \omega_{i}\left\|x_{i}\right\|^{2} \leq 1, y \in M^{\perp},\|y\| \leq 1
\end{array}\right\} \\
& \leq \sup \left\{\sum_{i=1}^{r} \omega_{i}\left\langle x_{i}, y\right\rangle: \begin{array}{l}
x_{i} \in M_{i}, i=1, \ldots, r, \sum_{i=1}^{r} \omega_{i}\left\|x_{i}\right\|^{2} \leq 1, \\
y \in M^{\perp},\|y\| \leq 1
\end{array}\right\} \\
& \leq \sup \left\{\sum_{i=1}^{r} \omega_{i}\left\langle x_{i}, y\right\rangle: \begin{array}{l}
x_{i} \in M_{i}, i=1, \ldots, r, \sum_{i=1}^{r} \omega_{i}\left\|P_{M_{i} \cap M^{\perp}}\left(x_{i}\right)\right\|^{2} \leq 1, \\
y \in M^{\perp},\|y\| \leq 1
\end{array}\right\} \\
& =\sup \left\{\sum_{i=1}^{r} \omega_{i}\left\langle z_{i}, y\right\rangle: \begin{array}{l}
z_{i} \in M_{i} \cap M^{\perp}, i=1, \ldots, r, \sum_{i=1}^{r} \omega_{i}\left\|z_{i}\right\|^{2} \leq 1, \\
y \in M^{\perp},\|y\| \leq 1
\end{array}\right\} \\
& =\sup \left\{\begin{array}{l}
\left.\langle\mathbf{z}, \mathbf{y}\rangle_{\omega}: \begin{array}{l}
\mathbf{z} \in \mathbf{C}_{\omega} \cap \mathbf{M}_{\omega}^{\perp},\|\mathbf{z}\|_{\omega} \leq 1, \\
\mathbf{y} \in \mathbf{D}_{\omega} \cap \mathbf{M}_{\omega}^{\perp},\|\mathbf{y}\|_{\omega} \leq 1
\end{array}\right\}=\alpha,
\end{array}\right.
\end{aligned}
$$

where in the first two inequalities, we take the supremum over a larger set. In the fourth line the equality holds since for every $\mathbf{x}=\left\{x_{i}\right\}_{i=0}^{r} \in \mathbf{H}_{\omega}$ such that $x_{i} \in M_{i}$ and $\sum_{i=1}^{r} \omega_{i}\left\|P_{M_{i} \cap M^{\perp}}\left(x_{i}\right)\right\|^{2} \leq 1$, there is at least one $\mathbf{z}=\left\{z_{i}\right\}_{i=0}^{r} \in \mathbf{H}_{\omega}$ with $z_{i} \in M_{i} \cap M^{\perp}$ and $\|\mathbf{z}\|_{\omega}^{2} \leq 1$ for which the equality $\sum_{i=1}^{r} \omega_{i}\left\langle x_{i}, y\right\rangle=$ $\sum_{i=1}^{r} \omega_{i}\left\langle z_{i}, y\right\rangle$ holds for all $y \in M^{\perp}$. For example, by (4.4), one can take $z_{i}:=P_{M_{i} \cap M^{\perp}}\left(x_{i}\right)$. This shows that $\cos _{\omega}\left(\mathbf{C}_{\omega}, \mathbf{D}_{\omega}\right)=\alpha$.

Lemma 4.2. Let $\omega \in \Omega_{r}$. The configuration constant between $\mathbf{C}_{\omega}$ and $\mathbf{D}_{\omega}$ satisfies:

$$
\begin{aligned}
& c_{\omega}\left(\mathbf{C}_{\omega}, \mathbf{D}_{\omega}\right)=\sup \left\{\langle\mathbf{x}, \mathbf{y}\rangle_{\omega}: \begin{array}{l}
\mathbf{x} \in \mathbf{C}_{\omega} \cap \mathbf{M}_{\omega}^{\perp} \cap \boldsymbol{\Delta}_{\omega}, \\
\mathbf{y} \in \mathbf{D}_{\omega} \cap \mathbf{M}_{\omega}^{\perp} \cap \boldsymbol{\Delta}_{\omega}
\end{array}\right\} \\
& =\sup \left\{\sum_{i=1}^{r} \omega_{i}\left\langle x_{i}, y\right\rangle: \begin{array}{l}
x_{i} \in M_{i} \cap M^{\perp},\left\|x_{i}\right\| \leq 1, i=1, \ldots, r \\
y \in M^{\perp},\|y\| \leq 1
\end{array}\right\} .
\end{aligned}
$$

Proof. The argument is similar to the one presented in the proof of Lemma 4.1, where one should write " $\sup _{i=1, \ldots, r}\|\cdot\| \leq 1$ " instead of " $\sum_{i=1}^{r} \omega_{i}\|\cdot\|^{2} \leq 1$ " in (4.6). We leave the details to the reader.

Lemma 4.3. Let $\omega \in \Omega_{r}$. The following estimates hold:

$$
\cos _{\omega}\left(\mathbf{C}_{\omega}, \mathbf{D}_{\omega}\right)^{2} \leq c_{\omega}\left(\mathbf{C}_{\omega}, \mathbf{D}_{\omega}\right) \leq \cos _{\omega}\left(\mathbf{C}_{\omega}, \mathbf{D}_{\omega}\right)
$$

Proof. By Theorem 3.3, we obtain

$$
\cos _{\omega}\left(\mathbf{C}_{\omega}, \mathbf{D}_{\omega}\right)^{2}=\left\|T_{\omega}-P_{M}\right\|=\sup \left\{\left\|\sum_{i=1}^{r} \omega_{i}\left(P_{M_{i}}(x)-P_{M}(x)\right)\right\|: x \in M^{\perp},\|x\| \leq 1\right\} .
$$

Note that, by (4.2), for all $x \in M^{\perp}$, we have

$$
P_{M_{i}}(x)-P_{M}(x)=P_{M_{i}}(x)=P_{M_{i}} P_{M^{\perp}}(x)=P_{M_{i} \cap M^{\perp}}(x) \in M_{i} \cap M^{\perp}
$$

and $\left\|P_{M_{i} \cap M^{\perp}}(x)\right\| \leq\|x\| \leq 1$. Consequently,

$$
\cos _{\omega}\left(\mathbf{C}_{\omega}, \mathbf{D}_{\omega}\right)^{2} \leq \sup \left\{\left\|\sum_{i=1}^{r} \omega_{i} x_{i}\right\|: x_{i} \in M_{i} \cap M^{\perp}, i=1, \ldots, r,\left\|x_{i}\right\| \leq 1\right\} .
$$


By the Riesz representation theorem, (2.6), the assumption that $x_{i} \in M_{i} \cap M^{\perp}$ and the fact that $\sum_{i=1}^{r} \omega_{i} x_{i} \in M^{\perp}$, we get

$$
\left\|\sum_{i=1}^{r} \omega_{i} x_{i}\right\|=\sup \left\{\sum_{i=1}^{r} \omega_{i}\left\langle x_{i}, y\right\rangle: y \in M^{\perp},\|y\| \leq 1\right\} \leq c_{\omega}\left(\mathbf{C}_{\omega}, \mathbf{D}_{\omega}\right) .
$$

This, when combined with (4.11), proves the first inequality in (4.8). The second inequality in (4.8) is trivial.

Remark 4.4. Let $\omega \in \Omega_{r}$ and assume that $J:=\left\{j: M_{j} \neq M\right\} \neq \emptyset$. Note that $M_{j} \cap M^{\perp} \neq\{0\}$ for all $j \in J$ and $M_{j} \cap M^{\perp}=\{0\}$ whenever $j \notin J$; compare with the proof of Proposition 2.4. It is not difficult to show that

$$
\begin{aligned}
& \cos _{\omega}\left(\mathbf{C}_{\omega}, \mathbf{D}_{\omega}\right)=\sup \left\{\begin{array}{ll}
\sum_{j \in J} \omega_{j}\left\langle x_{j}, y\right\rangle: \begin{array}{l}
x_{j} \in M_{j} \cap M^{\perp}, j \in J, \sum_{j \in J} \omega_{j}\left\|x_{j}\right\|^{2}=1, \\
y \in M^{\perp},\|y\|=1
\end{array}
\end{array}\right\} \\
& =\sup \left\{\left\|\sum_{j \in J} \omega_{j} x_{j}\right\|: x_{j} \in M_{j} \cap M^{\perp}, j \in J, \sum_{j \in J} \omega_{j}\left\|x_{j}\right\|^{2}=1\right\} \\
& =\sup \left\{\frac{\left\|\sum_{j \in J} \omega_{j} x_{j}\right\|}{\sqrt{\sum_{j \in J} \omega_{j}\left\|x_{j}\right\|^{2}}}: x_{j} \in M_{j} \cap M^{\perp}, j \in J, 0 \neq \sum_{j \in J} \omega_{j}\left\|x_{j}\right\|^{2}<\infty\right\}
\end{aligned}
$$

and

$$
\begin{aligned}
& c_{\omega}\left(\mathbf{C}_{\omega}, \mathbf{D}_{\omega}\right)=\sup \left\{\begin{array}{ll}
\sum_{j \in J} \omega_{j}\left\langle x_{j}, y\right\rangle: & \begin{array}{l}
x_{j} \in M_{j} \cap M^{\perp},\left\|x_{j}\right\|=1, j \in J, \\
y \in M^{\perp},\|y\|=1
\end{array}
\end{array}\right\} \\
& =\sup \left\{\left\|\sum_{j \in J} \omega_{j} x_{j}\right\|: x_{j} \in M_{j} \cap M^{\perp},\left\|x_{j}\right\|=1, j \in J\right\} \text {. }
\end{aligned}
$$

Theorem 4.5 (Reduction to Unique Subspaces). Let $q \in \mathbb{Z}_{+} \cup\{\infty\}$ be such that $q \leq r$, let $L_{j}$ be nontrivial, closed and linear subspaces of $\mathcal{H}, j=1, \ldots, q$, and let $L$ be their intersection. Moreover, let $\left\{I_{j}\right\}_{j=1}^{q}$ consist of nonempty, pairwise disjoint subsets of $\{1, \ldots, r\}$, possibly infinite, such that $\bigcup_{j=1}^{q} I_{j}=\{1, \ldots, r\}$, and assume that $M_{i}=L_{j}$ for all $i \in I_{j}$. Then,

$$
\cos _{\omega}\left(\mathbf{C}_{\omega}, \mathbf{D}_{\omega}\right)=\cos _{\lambda}\left(\mathbf{E}_{\lambda}, \mathbf{D}_{\lambda}\right) \quad \text { and } \quad c_{\omega}\left(\mathbf{C}_{\omega}, \mathbf{D}_{\omega}\right)=c_{\lambda}\left(\mathbf{E}_{\lambda}, \mathbf{D}_{\lambda}\right),
$$

where $\lambda:=\left\{\lambda_{j}\right\}_{j=1}^{q} \in \Omega_{q}$ with $\lambda_{j}:=\sum_{i \in I_{j}} \omega_{i}$ and where (compare with (2.11))

$$
\mathbf{E}_{\lambda}:= \begin{cases}\prod_{j=1}^{q} L_{j}, & \text { if } q \in \mathbb{Z}_{+} \\ \left\{\left\{u_{j}\right\}_{j=1}^{\infty}: u_{j} \in L_{j}, j=1,2, \ldots, \sum_{j=1}^{\infty} \lambda_{j}\left\|u_{j}\right\|^{2}<\infty\right\}, & \text { if } q=\infty .\end{cases}
$$

Proof. We demonstrate only the first equality in (4.18) by using Lemma 4.1. A similar argument, when combined with Lemma 4.2, can be used to establish the second equality in (4.18).

In order to show the inequality $\cos _{\omega}\left(\mathbf{C}_{\omega}, \mathbf{D}_{\omega}\right) \leq \cos _{\lambda}\left(\mathbf{E}_{\lambda}, \mathbf{D}_{\lambda}\right)$, for each pair of points

$$
\mathbf{x}=\left\{x_{i}\right\}_{i=1}^{r} \in \mathbf{C}_{\omega} \cap \mathbf{M}_{\omega}^{\perp} \cap \mathbf{B}_{\omega} \quad \text { and } \quad \mathbf{y}=\{y\}_{i=1}^{r} \in \mathbf{D}_{\omega} \cap \mathbf{M}_{\omega}^{\perp} \cap \mathbf{B}_{\omega}
$$


in $\mathbf{H}_{\omega}$ we define another pair

$$
\mathbf{u}=\left\{u_{j}\right\}_{j=1}^{q} \in \mathbf{E}_{\lambda} \cap \mathbf{L}_{\lambda}^{\perp} \cap \mathbf{B}_{\lambda} \quad \text { and } \quad \mathbf{v}=\{v\}_{j=1}^{q} \in \mathbf{D}_{\lambda} \cap \mathbf{L}_{\lambda}^{\perp} \cap \mathbf{B}_{\lambda}
$$

in $\mathbf{H}_{\lambda}$ which satisfies

$$
\langle\mathbf{x}, \mathbf{y}\rangle_{\omega}=\langle\mathbf{u}, \mathbf{v}\rangle_{\lambda},
$$

where analogously to (2.13),

$$
\mathbf{L}_{\lambda}:= \begin{cases}\prod_{j=1}^{q} L, & \text { if } q \in \mathbb{Z}_{+} \\ \left\{\left\{u_{j}\right\}_{j=1}^{\infty}: u_{j} \in L, j=1,2, \ldots, \sum_{j=1}^{\infty} \lambda_{j}\left\|u_{j}\right\|^{2}<\infty\right\}, & \text { if } q=\infty .\end{cases}
$$

To this end, for each $j \in\{1, \ldots, q\}$, define $u_{j}:=\frac{1}{\lambda_{j}} \sum_{i \in I_{j}} \omega_{i} x_{i}$. Notice that $u_{j}$ is well defined and $u_{j} \in L_{j}$. Moreover, we have $\sum_{i=1}^{r} \omega_{i} x_{i}=\sum_{j=1}^{q} \lambda_{j} u_{j}$, where for $r=\infty$ we use Lemma 6.1. By the convexity of $\|\cdot\|^{2}$

$$
\sum_{j=1}^{q} \lambda_{j}\left\|u_{j}\right\|^{2}=\sum_{j=1}^{q} \lambda_{j}\left\|\sum_{i \in I_{j}} \frac{\omega_{i}}{\lambda_{j}} x_{i}\right\|^{2} \leq \sum_{j=1}^{q} \lambda_{j} \sum_{i \in I_{j}} \frac{\omega_{i}}{\lambda_{j}}\left\|x_{i}\right\|^{2}=\|\mathbf{x}\|_{\omega}^{2}<\infty,
$$

that is, $\mathbf{u} \in \mathbf{H}_{\lambda}$. On the other hand, since $L=M$, we can define $v:=y$. It is not difficult to see that with the above defined $\mathbf{u}$ and $\mathbf{v}$, equality (4.22) holds.

In order to prove the opposite inequality $\cos _{\omega}\left(\mathbf{C}_{\omega}, \mathbf{D}_{\omega}\right) \geq \cos _{\lambda}\left(\mathbf{E}_{\lambda}, \mathbf{D}_{\lambda}\right)$, this time for each pair in (4.21) we define the corresponding pair in (4.20), for which again equality (4.22) holds. It suffices to take $x_{i}:=u_{j}$ for all $i \in I_{j}$ and $y:=v$. Indeed, by assumption, $x_{i} \in M_{i}$. Moreover, $\sum_{i \in I_{j}} \omega_{i} x_{i}=\lambda_{j} u_{j}$, hence $\sum_{i=1}^{r} \omega_{i} x_{i}=\sum_{j=1}^{q} \lambda_{j} u_{j}$. Furthermore,

$$
\sum_{i=1}^{r} \omega_{i}\left\|x_{i}\right\|^{2}=\sum_{j=1}^{q} \lambda_{j}\left\|u_{j}\right\|^{2}=\|\mathbf{u}\|_{\lambda}^{2}<\infty
$$

which shows that $\mathbf{x} \in \mathbf{H}_{\omega}$. Clearly, equality (4.22) holds for the pair (x,y) defined above. This completes the proof.

Theorem 4.6 (Approximation, $r=\infty$ ). Let $\omega \in \Omega_{\infty}$. Then

$$
\cos _{\omega}\left(\mathbf{C}_{\omega}, \mathbf{D}_{\omega}\right)=\lim _{q \rightarrow \infty} \cos _{\lambda_{q}}\left(\mathbf{C}_{\lambda_{q}}, \mathbf{D}_{\lambda_{q}}\right) \quad \text { and } \quad c_{\omega}\left(\mathbf{C}_{\omega}, \mathbf{D}_{\omega}\right)=\lim _{q \rightarrow \infty} c_{\lambda_{q}}\left(\mathbf{C}_{\lambda_{q}}, \mathbf{D}_{\lambda_{q}}\right),
$$

where $\lambda_{q}:=\left\{\frac{\omega_{1}}{s_{q}}, \ldots, \frac{\omega_{q}}{s_{q}}\right\} \in \Omega_{q}$ with $s_{q}:=\sum_{i=1}^{q} \omega_{i}$ and $q=2,3, \ldots$.

Proof. We only show the first equality in (4.26). A similar argument can be employed to prove the second equality.

Note that for all $q=2,3, \ldots$, we have $s_{q}<s_{q+1}<1, N_{q}^{\perp} \subset N_{q+1}^{\perp} \subset M^{\perp}$, where $N_{q}:=\bigcap_{i=1}^{q} M_{i}$ and

$$
\mathbf{C}_{\lambda_{q}} \cap \mathbf{M}_{\lambda_{q}}^{\perp} \cap \mathbf{B}_{\lambda_{q}}=\left\{\left\{\begin{array}{ll}
\left\{x_{i}\right\}_{i=1}^{q}: & \begin{array}{l}
x_{i} \in M_{i} \cap N_{q}^{\perp}, i=1, \ldots, q \\
\sum_{i=1}^{q} \omega_{i}\left\|x_{i}\right\|^{2} \leq s_{q}
\end{array}
\end{array}\right\}\right.
$$

Consequently, if $\left\{x_{1}, \ldots, x_{q}\right\} \in \mathbf{C}_{\lambda_{q}} \cap \mathbf{M}_{\lambda_{q}}^{\perp} \cap \mathbf{B}_{\lambda_{q}}$, then $\left\{x_{1}, \ldots, x_{q}, 0\right\} \in \mathbf{C}_{\lambda_{q+1}} \cap \mathbf{M}_{\lambda_{q+1}}^{\perp} \cap \mathbf{B}_{\lambda_{q+1}}$ and analogously $\left\{x_{1}, \ldots, x_{q}, 0,0, \ldots\right\} \in \mathbf{C}_{\omega} \cap \mathbf{M}_{\omega}^{\perp} \cap \mathbf{B}_{\omega}$. Hence, by Lemma 4.1,

$$
s_{q} \cos _{\lambda_{q}}\left(\mathbf{C}_{\lambda_{q}}, \mathbf{D}_{\lambda_{q}}\right) \leq s_{q+1} \cos _{\lambda_{q+1}}\left(\mathbf{C}_{\lambda_{q+1}}, \mathbf{D}_{\lambda_{q+1}}\right) \leq \cos _{\omega}\left(\mathbf{C}_{\omega}, \mathbf{D}_{\omega}\right)
$$


and thus the sequence $\left\{s_{q} \cos _{\lambda_{q}}\left(\mathbf{C}_{\lambda_{q}}, \mathbf{D}_{\lambda_{q}}\right)\right\}_{q=2}^{\infty}$ is monotone and bounded, and therefore converges to some number $\alpha$. Moreover, $\alpha \leq \cos _{\omega}\left(\mathbf{C}_{\omega}, \mathbf{D}_{\omega}\right)$.

In order to show the opposite inequality $\alpha \geq \cos _{\omega}\left(\mathbf{C}_{\omega}, \mathbf{D}_{\omega}\right)$, we first demonstrate that for each pair

$$
\mathbf{x}=\left\{x_{i}\right\}_{i=1}^{\infty} \in \mathbf{C}_{\omega} \cap \mathbf{M}_{\omega}^{\perp} \cap \mathbf{B}_{\omega} \quad \text { and } \quad \mathbf{y}=\{y\}_{i=1}^{\infty} \in \mathbf{C}_{\omega} \cap \mathbf{M}_{\omega}^{\perp} \cap \mathbf{B}_{\omega}
$$

in $\mathbf{H}_{\omega}$ and for each $\varepsilon>0$, we can find another pair

$$
\mathbf{x}_{q}=\left\{x_{q, i}\right\}_{i=1}^{q} \in \mathbf{C}_{\lambda_{q}} \cap \mathbf{M}_{\lambda_{q}}^{\perp} \cap \mathbf{B}_{\lambda_{q}} \quad \text { and } \quad \mathbf{y}_{q}=\left\{y_{q}\right\}_{i=1}^{q} \in \mathbf{C}_{\lambda_{q}} \cap \mathbf{M}_{\lambda_{q}}^{\perp} \cap \mathbf{B}_{\lambda_{q}}
$$

in $\mathbf{H}_{\lambda_{q}}$ such that

$$
\langle\mathbf{x}, \mathbf{y}\rangle_{\omega} \leq s_{q}\left\langle\mathbf{x}_{q}, \mathbf{y}_{q}\right\rangle_{\lambda_{q}}+\varepsilon .
$$

To this end, choose $n$ so that the tail satisfies $\sum_{i=n+1}^{\infty} \omega_{i}\left\langle x_{i}, y\right\rangle<\frac{\varepsilon}{2}$. For each $q>n$, define

$$
x_{q, i}:=\left\{\begin{array}{ll}
\sqrt{s_{q}} \cdot P_{M_{i} \cap N_{q}^{\perp}}\left(x_{i}\right), & \text { if } i=1, \ldots, n \\
0, & \text { otherwise }
\end{array} \text { and } y_{q}:=\sqrt{s_{q}} \cdot P_{N_{q}^{\perp}}(y) .\right.
$$

Note that $\mathbf{x}_{q}$ and $\mathbf{y}_{q}$ indeed satisfy (4.30) as $\left\|\mathbf{x}_{q}\right\|_{\lambda_{q}} \leq 1$ and $\left\|\mathbf{y}_{q}\right\| \leq 1$.

On the other hand, the decreasing sequence of sets $\left\{N_{q}\right\}_{q=2}^{\infty}$ converges to $M$ in the sense of Mosco; see [28, Definition 1.2 and Lemma 3.1]. This, when combined with [35, Theorem 3.2], implies that for all $x \in \mathcal{H}$, we get $P_{N_{q}}(x) \rightarrow P_{M}(x)$ as $q \rightarrow \infty$ and further, $P_{N_{q}}(x)=x-P_{N_{q}}(x) \rightarrow x-P_{M}(x)=P_{M^{\perp}}(x)$ as $q \rightarrow \infty$. In this connection, see also [24, Proposition 7] and [9, Lemma 4.2].

In particular, using the assumptions that $x_{i} \in M_{i} \cap M^{\perp}, y \in M^{\perp}$ and the equality $P_{M_{i} \cap N_{q}^{\perp}}=P_{N_{q}^{\perp}} P_{M_{i}}$ (compare with (4.2)), we obtain

$$
x_{q, i} \rightarrow P_{M^{\perp}}\left(x_{i}\right)=x_{i} \quad \text { and } \quad y_{q} \rightarrow P_{M^{\perp}}(y)=y
$$

as $q \rightarrow \infty$. Consequently, for all large enough $q$ and for all $i=1, \ldots, n$, we reach the inequality $\left\langle x_{i}, y\right\rangle \leq\left\langle x_{q, i}, y_{q}\right\rangle+\frac{\varepsilon}{2 n}$, which leads to

$$
\langle\mathbf{x}, \mathbf{y}\rangle_{\omega} \leq \sum_{i=1}^{n} \omega_{i}\left\langle x_{i}, y\right\rangle+\frac{\varepsilon}{2} \leq \sum_{i=1}^{n} \omega_{i}\left\langle x_{q, i}, y_{q}\right\rangle+\varepsilon=s_{q}\left\langle\mathbf{x}_{q}, \mathbf{y}_{q}\right\rangle_{\lambda_{q}}+\varepsilon .
$$

This shows (4.31), as claimed.

We are now ready to return to the inequality $\alpha \geq \cos _{\omega}\left(\mathbf{C}_{\omega}, \mathbf{D}_{\omega}\right)$. Indeed, by (4.31), and by the monotonicity of the sequence $\left\{s_{q} \cos _{\lambda_{q}}\left(\mathbf{C}_{\lambda_{q}}, \mathbf{D}_{\lambda_{q}}\right)\right\}_{q=2}^{\infty}$, we have

$$
\langle\mathbf{x}, \mathbf{y}\rangle_{\omega} \leq s_{q}\left\langle\mathbf{x}_{q}, \mathbf{y}_{q}\right\rangle_{\lambda_{q}}+\varepsilon \leq s_{q} \cos _{\lambda_{q}}\left(\mathbf{C}_{\lambda_{q}}, \mathbf{D}_{\lambda_{q}}\right)+\varepsilon \leq \alpha+\varepsilon .
$$

By taking the supremum over all $\mathbf{x}$ and $\mathbf{y}$ satisfying (4.29), we obtain that $\cos _{\omega}\left(\mathbf{C}_{\omega}, \mathbf{D}_{\omega}\right) \leq \alpha+\varepsilon$, which proves the asserted inequality and hence completes the proof.

Proposition 4.7. Let $\omega \in \Omega_{r}$ and assume that the subspaces $M_{1}, \ldots, M_{r}$ are nontrivial and pairwise orthogonal. Then

$$
c_{\omega}\left(\mathbf{C}_{\omega}, \mathbf{D}_{\omega}\right)=\sqrt{\sum_{i=1}^{r} \omega_{i}^{2}} \quad \text { and } \quad \cos _{\omega}\left(\mathbf{C}_{\omega}, \mathbf{D}_{\omega}\right)=\sqrt{\sup _{i=1, \ldots, r} \omega_{i}} .
$$


Proof. Observe first that we must have $J:=\left\{j: M_{j} \neq M\right\}=\{1, \ldots, r\}$. Otherwise, there would be a pair $i$ and $j$ for which $M_{i}=M \subset M_{j}$ and $M_{i} \perp M_{j}$, which is possible only when $M_{i}=\{0\}$, a contradiction. Moreover, $\sup _{i} \omega_{i}=\omega_{j}$ for some $j \in\{1, \ldots, r\}$, even when $r=\infty$. By the assumed pairwise orthogonality, for all $\left\{x_{i}\right\}_{i=1}^{r}$ with $x_{i} \in M_{i} \cap M^{\perp}$ and $\sum_{i=1}^{r} \omega_{i}\left\|x_{i}\right\|^{2}=1$, we have

$$
\left\|\sum_{i=1}^{r} \omega_{i} x_{i}\right\|^{2}=\sum_{i=1}^{r} \omega_{i}^{2}\left\|x_{i}\right\|^{2} \leq \omega_{j} \sum_{i=1}^{r} \omega_{i}\left\|x_{i}\right\|^{2}=\omega_{j} .
$$

Thus, by (4.14), $\cos _{\omega}\left(\mathbf{C}_{\omega}, \mathbf{D}_{\omega}\right) \leq \omega_{j}$. On the other hand, when $\left\|x_{j}\right\|=\frac{1}{\sqrt{\omega_{j}}}$, the assumption $\sum_{i=1}^{\infty} \omega_{i}\left\|x_{i}\right\|^{2}=$ 1 implies that $\left\|x_{i}\right\|=0$ for all $i \neq j$. Hence, in this case, $\left\|\sum_{i=1}^{r} \omega_{i} x_{i}\right\|^{2}=\omega_{j}$ and therefore $\cos _{\omega}\left(\mathbf{C}_{\omega}, \mathbf{D}_{\omega}\right)=$ $\omega_{j}$. In view of (4.17) and (4.37), it is not difficult to see that $c_{\omega}\left(\mathbf{C}_{\omega}, \mathbf{D}_{\omega}\right)=\sqrt{\sum_{i=1}^{r} \omega_{i}^{2}}$.

In the next example, we show that the equality or inequality between $c_{\omega}\left(\mathbf{C}_{\omega}, \mathbf{D}_{\omega}\right)$ and $\cos _{\omega}\left(\mathbf{C}_{\omega}, \mathbf{D}_{\omega}\right)$ may depend on the weights $\omega \in \Omega_{r}$.

Example 4.8. Let $q \in \mathbb{Z}_{+}$be such that $q \leq r$ and let $L_{1}, \ldots, L_{q}$ be a tuple of nontrivial, closed and linear subspaces of $\mathcal{H}$, which are pairwise orthogonal. Assume that the list $M_{1}, \ldots, M_{r}$ consists only of subspaces from $L_{1}, \ldots, L_{q}$ and let $\lambda \in \Omega_{q}$ be defined as in Theorem 4.5. Then, by Theorem 4.5 and Proposition 4.7, we have $c_{\omega}\left(\mathbf{C}_{\omega}, \mathbf{D}_{\omega}\right)=\sqrt{\sum_{j=1}^{q} \lambda_{j}^{2}}$ and $\cos _{\omega}\left(\mathbf{C}_{\omega}, \mathbf{D}_{\omega}\right)=\sqrt{\sup _{j=1, \ldots, q} \lambda_{j}}$. We consider two cases:

(a) If $\lambda_{j}=1 / q$ for all $j=\{1, \ldots, q\}$, then $c_{\omega}\left(\mathbf{C}_{\omega}, \mathbf{D}_{\omega}\right)=\cos _{\omega}\left(\mathbf{C}_{\omega}, \mathbf{D}_{\omega}\right)=\sqrt{1 / q}$.

(b) If $\lambda_{i}:=\max _{j=1, \ldots, q} \lambda_{j}>1 / q$, then $\sum_{j=1}^{q} \lambda_{j}^{2}<\lambda_{i} \sum_{j=1}^{q} \lambda_{j}=\lambda_{i}$ and consequently, $c_{\omega}\left(\mathbf{C}_{\omega}, \mathbf{D}_{\omega}\right)<$ $\cos _{\omega}\left(\mathbf{C}_{\omega}, \mathbf{D}_{\omega}\right)$.

In spite of the previous example, the "parallel" alignment of the subspaces $M_{i}$ does not depend on $\omega$, as we show in our next theorem.

Theorem 4.9. The following conditions are equivalent:

(i) There is $\lambda=\left\{\lambda_{i}\right\}_{i=1}^{r} \in \Omega_{r}$ such that $\cos _{\lambda}\left(\mathbf{C}_{\lambda}, \mathbf{D}_{\lambda}\right)=1$.

(ii) For all $\omega=\left\{\omega_{i}\right\}_{i=1}^{r} \in \Omega_{r}$, we have $\cos _{\omega}\left(\mathbf{C}_{\omega}, \mathbf{D}_{\omega}\right)=1$.

Proof. Assume that $\cos _{\lambda}\left(\mathbf{C}_{\lambda}, \mathbf{D}_{\lambda}\right)=1$. It suffices to show that there is at least one sequence of pairs $\left\{\mathbf{x}_{k}, \mathbf{y}_{k}\right\}_{k=1}^{\infty}$, which for all $\omega \in \Omega_{r}$ satisfies

$$
\mathbf{x}_{k}=\left\{x_{k, i}\right\}_{i=1}^{r} \in \mathbf{C}_{\omega} \cap \mathbf{M}_{\omega}^{\perp} \cap \mathbf{B}_{\omega}, \quad \mathbf{y}_{k}=\left\{y_{k}\right\}_{i=1}^{r} \in \mathbf{D}_{\omega} \cap \mathbf{M}_{\omega}^{\perp} \cap \mathbf{B}_{\omega}
$$

and

$$
\lim _{k \rightarrow \infty}\left\langle\mathbf{x}_{k}, \mathbf{y}_{k}\right\rangle_{\omega}=1
$$

By Lemma 4.3, $c_{\lambda}\left(\mathbf{C}_{\lambda}, \mathbf{D}_{\lambda}\right)=1$. Observe that we must have $J:=\left\{j: M_{j} \neq M\right\}=\{1, \ldots, r\}$. Otherwise, by (4.16) and by the Cauchy-Schwarz inequality (applied to each summand), we would arrive at the following contradiction: $1=c_{\lambda}\left(\mathbf{C}_{\lambda}, \mathbf{D}_{\lambda}\right) \leq \sum_{j \in J} \lambda_{j}<1$. Consequently, by the definition of the supremum in (4.16), for each $k=1,2, \ldots$, there are

$$
\mathbf{x}_{k}=\left\{x_{k, i}\right\}_{i=1}^{r}, \quad x_{k, i} \in M_{i} \cap M^{\perp}, \quad\left\|x_{k, i}\right\|=1
$$


and

$$
\mathbf{y}_{k}=\left\{y_{k}\right\}_{i=1}^{r}, \quad y_{k} \in M^{\perp}, \quad\left\|y_{k}\right\|=1,
$$

which satisfy

$$
1-\frac{1}{k} \leq\left\langle\mathbf{x}_{k}, \mathbf{y}_{k}\right\rangle_{\lambda}=\sum_{i=1}^{r} \lambda_{i}\left\langle x_{k, i}, y_{k}\right\rangle \leq 1
$$

Without any loss to the generality we may assume that $\left\langle x_{k, i}, y_{k}\right\rangle \geq 0$ for all $i=1, \ldots, r$ and all $k=1,2, \ldots$. Indeed, if $\left\langle x_{k, i}, y_{k}\right\rangle<0$ for some $k$ and $i$ then, by replacing " $x_{k, i}$ " by " $-x_{k, i}$ ", we can only increase the number $\left\langle\mathbf{x}_{k}, \mathbf{y}_{k}\right\rangle_{\lambda}$ in (4.42).

Obviously, the above-defined sequence of pairs $\left\{\mathbf{x}_{k}, \mathbf{y}_{k}\right\}_{k=1}^{\infty}$ satisfies (4.38) for all $\omega \in \Omega_{r}$ and (4.39) for $\omega=\lambda$. What remains to be shown is that $\left\{\mathbf{x}_{k}, \mathbf{y}_{k}\right\}_{k=1}^{\infty}$ satisfies (4.39) for all $\omega \in \Omega_{r}$. Before doing so, we investigate the properties of $\left\langle x_{k, i}, y_{k}\right\rangle$ in more detail.

Note that by the Cauchy-Schwarz inequality, we have $\left\langle x_{k, i}, y_{k}\right\rangle \leq 1$. We now show that for each $i=1, \ldots, r$, we have

$$
\lim _{k \rightarrow \infty}\left\langle x_{k, i}, y_{k}\right\rangle=1
$$

Suppose to the contrary that

$$
\liminf _{k \rightarrow \infty}\left\langle x_{k, j}, y_{k}\right\rangle=\lim _{n \rightarrow \infty}\left\langle x_{k_{n}, j}, y_{k_{n}}\right\rangle=1-\varepsilon
$$

for some $j$ and some $\varepsilon \in(0,1]$, where $\left\{k_{n}\right\}_{n=1}^{\infty}$ is a subsequence of $\{k\}_{k=1}^{\infty}$. By taking $n$ large enough, we may assume that $\left\langle x_{k_{n}, j}, y_{k_{n}}\right\rangle \leq 1-\frac{\varepsilon}{2}$. This, when combined with (4.42), leads to

$$
1-\frac{1}{k_{n}} \leq \lambda_{j}\left\langle x_{k_{n}, j}, y_{k_{n}}\right\rangle+\sum_{i \neq j} \lambda_{i}\left\langle x_{k_{n}, i}, y_{k_{n}}\right\rangle \leq \lambda_{j}\left(1-\frac{\varepsilon}{2}\right)+\sum_{i \neq j} \lambda_{i}=1-\lambda_{j} \frac{\varepsilon}{2}<1,
$$

which is a contradiction since the left-hand side converges to one as $n \rightarrow \infty$.

We are now ready to show that the above-defined sequence of pairs $\left\{\mathbf{x}_{k}, \mathbf{y}_{k}\right\}_{k=1}^{\infty}$ satisfies (4.39) for all $\omega \in \Omega_{r}$. Indeed, let $\omega \in \Omega_{r}$. Moreover, let $\varepsilon \in(0,1)$ and let $n \in\{1, \ldots, r\}$ be an integer such that $\sum_{i=1}^{n} \omega_{i} \geq \sqrt{1-\varepsilon}$. Obviously, when $r \in \mathbb{Z}_{+}$, we can take $n:=r$. By (4.43), we may assume that $\left\langle x_{k, i}, y_{k}\right\rangle \geq \sqrt{1-\varepsilon}$ for all $i=1,2, \ldots, n$ and all large enough $k \geq K_{n}$. Thus, for all $k \geq K_{n}$, we arrive at

$$
1 \geq\left\langle\mathbf{x}_{k}, \mathbf{y}_{k}\right\rangle_{\omega} \geq \sum_{i=1}^{n} \omega_{i}\left\langle x_{k, i}, y_{k}\right\rangle \geq \sum_{i=1}^{n} \omega_{i} \sqrt{1-\varepsilon} \geq 1-\varepsilon
$$

which shows that $\left\langle\mathbf{x}_{k}, \mathbf{y}_{k}\right\rangle_{\omega} \rightarrow 1$ as $k \rightarrow \infty$. This proves (4.39) and completes the proof of the lemma itself.

Remark 4.10 (Erratum to [33]). As we have already observed in Proposition 2.4, $\mathbf{C}_{\omega} \cap \mathbf{M}_{\omega}^{\perp}$ may be a proper subset of $\mathbf{C}_{\omega} \cap\left(\mathbf{C}_{\omega} \cap \mathbf{D}_{\omega}\right)^{\perp}$ and equality need not hold. Consequently, the argument used in the proof of [33, Theorem 8] preceding [33, equality (19)] was incorrect. However, Lemma 4.1 justifies the validity of [33, equality (19)] because

$$
\cos _{\omega}\left(\mathbf{C}_{\omega}, \mathbf{D}_{\omega}\right)=\sup \left\{\frac{\langle\mathbf{x}, \mathbf{y}\rangle_{\omega}}{\|\mathbf{x}\|_{\omega}\|\mathbf{y}\|_{\omega}}: \begin{array}{l}
\mathbf{x} \in \mathbf{C}_{\omega} \cap \mathbf{M}_{\omega}^{\perp}, \mathbf{x} \neq \mathbf{0} \\
\mathbf{y} \in \mathbf{D}_{\omega} \cap \mathbf{M}_{\omega}^{\perp}, \mathbf{y} \neq \mathbf{0}
\end{array}\right\} .
$$




\section{Asymptotic Properties of the Simultaneous Projection Method}

In this section we oftentimes refer to the subspace $A_{\omega}\left(\mathbf{C}_{\omega}^{\perp}\right)$, the explicit form of which is given in Proposition 2.5. We begin with a theorem which corresponds to equivalence (1.5).

Theorem 5.1. Let $\omega \in \Omega_{r}$. The following conditions are equivalent:

(i) $\left\|T_{\omega}-P_{M}\right\|<1$;

(ii) $\cos _{\omega}\left(\mathbf{C}_{\omega}, \mathbf{D}_{\omega}\right)<1$;

(iii) The set $A_{\omega}\left(\mathbf{C}_{\omega}^{\perp}\right)$ is closed in $\mathcal{H}$; (iv) $\left\|P_{\mathbf{D}_{\omega}} P_{\mathbf{C}_{\omega}}-P_{\mathbf{C}_{\omega} \cap \mathbf{D}_{\omega}}\right\|_{\omega}<1$

(v) $\left\{\mathbf{C}_{\omega}, \mathbf{D}_{\omega}\right\}$ is linearly regular;

(vi) $\mathbf{C}_{\omega}^{\perp}+\mathbf{D}_{\omega}^{\perp}$ is closed in $\mathbf{H}_{\omega}$.

Proof. By (1.5) applied to $\mathbf{C}_{\omega}$ and $\mathbf{D}_{\omega}$, we have the equivalence between (ii) and (vi). Similarly, by [9, Theorem 5.19] applied to $\mathbf{C}_{\omega}$ and $\mathbf{D}_{\omega}$, we obtain the equivalence between (v) and (vi). Recall that $\left\{\mathbf{C}_{\omega}, \mathbf{D}_{\omega}\right\}$ is said to be linearly regular if the inequality $\max \left\{d\left(\mathbf{x}, \mathbf{C}_{\omega}\right), d\left(\mathbf{x}, \mathbf{D}_{\omega}\right)\right\} \leq \kappa d\left(\mathbf{x}, \mathbf{C}_{\omega} \cap \mathbf{D}_{\omega}\right)$ holds for all $\mathbf{x} \in \mathbf{H}_{\omega}$ and some $\kappa>0$. Proposition 2.5 verifies the equivalence between (iii) and (vi). Finally, by Theorems 3.3 and 1.5 , we have

$$
\left\|T_{\omega}-P_{M}\right\|=\cos _{\omega}\left(\mathbf{C}_{\omega}, \mathbf{D}_{\omega}\right)^{2}=\left\|P_{\mathbf{D}_{\omega}} P_{\mathbf{C}_{\omega}}-P_{\mathbf{C}_{\omega} \cap \mathbf{D}_{\omega}}\right\|_{\omega}
$$

which explains the equivalence between (i), (ii) and (iv).

Theorem 5.2 (Dichotomy). Exactly one of the following two statements holds:

(i) For all $\omega \in \Omega_{r}$, the set $A_{\omega}\left(\mathbf{C}_{\omega}^{\perp}\right)$ is closed in $\mathcal{H}$. Then the sequence $\left\{T_{\omega}^{k}\right\}_{k=1}^{\infty}$ converges linearly to $P_{M}$ as $k \rightarrow \infty$ and the optimal error bound is given by

$$
\left\|T_{\omega}^{k}(x)-P_{M}(x)\right\| \leq \cos _{\omega}\left(\mathbf{C}_{\omega}, \mathbf{D}_{\omega}\right)^{2 k} \cdot\|x\| .
$$

(ii) For all $\omega \in \Omega_{r}$, the set $A_{\omega}\left(\mathbf{C}_{\omega}^{\perp}\right)$ is not closed in $\mathcal{H}$. Then the sequence $\left\{T_{\omega}^{k}\right\}_{k=1}^{\infty}$ converges arbitrarily slowly to $P_{M}$ as $k \rightarrow \infty$.

Proof. By combining Theorems 4.9 and 5.1, we see that either $A_{\omega}\left(\mathbf{C}_{\omega}^{\perp}\right)$ is closed for all $\omega \in \Omega_{r}$ or $A_{\omega}\left(\mathbf{C}_{\omega}^{\perp}\right)$ is not closed for all $\omega \in \Omega_{r}$. This shows the dichotomy between (i) and (ii).

If we assume as in (i) that $A_{\omega}\left(\mathbf{C}_{\omega}^{\perp}\right)$ is closed, then both the linear convergence and the optimality of the estimate (5.2) follow from Theorems 3.3 and 5.1.

Assume now that $A_{\omega}\left(\mathbf{C}_{\omega}^{\perp}\right)$ is not closed, where $\omega \in \Omega_{r}$. We show that the sequence $\left\{T_{\omega}^{k}\right\}_{k=1}^{\infty}$ converges arbitrarily slowly to $P_{M}$ as $k \rightarrow \infty$. To this end, let $\left\{a_{k}\right\}_{k=1}^{\infty} \subset[0, \infty)$ be a null sequence and let $\left\{b_{k}\right\}_{k=1}^{\infty}$ be defined by $b_{1}:=a_{1}, b_{2}:=a_{1}$ and $b_{k}:=a_{k-1}, k \geq 3$. By Theorem 5.1, we see that $\mathbf{C}_{\omega}^{\perp}+\mathbf{D}_{\omega}^{\perp}$ is not closed in $\mathbf{H}_{\omega}$. This, when combined with Theorem 1.2, implies that the sequence $\left\{\left(P_{\mathbf{D}_{\omega}} P_{\mathbf{C}_{\omega}}\right)^{k}\right\}_{k=1}^{\infty}$ converges to $P_{\mathbf{C}_{\omega} \cap \mathbf{D}_{\omega}}$ arbitrarily slowly as $k \rightarrow \infty$. In particular, there is $\mathbf{x} \in \mathbf{H}_{\omega}$, such that

$$
\left\|\left(P_{\mathbf{D}_{\omega}} P_{\mathbf{C}_{\omega}}\right)^{k}(\mathbf{x})-P_{\mathbf{C}_{\omega} \cap \mathbf{D}_{\omega}}(\mathbf{x})\right\| \geq b_{k}
$$

for all $k=1,2, \ldots$. Note that $\mathbf{y}:=P_{\mathbf{D}_{\omega}} P_{\mathbf{C}_{\omega}}(\mathbf{x}) \in \mathbf{D}_{\omega}$, hence $\mathbf{y}=\{y\}_{i=1}^{r}$ for some $y \in \mathcal{H}$. Moreover, $P_{\mathbf{C}_{\omega} \cap \mathbf{D}_{\omega}}(\mathbf{x})=P_{\mathbf{C}_{\omega} \cap \mathbf{D}_{\omega}}(\mathbf{y})$ (compare with (3.16)). Consequently, by rewriting (5.3) in terms of $\mathbf{y}$ and $a_{k}$, and by Theorem 3.2, we arrive at

$$
\left\|T_{\omega}^{k}(y)-P_{M}(y)\right\|=\left\|\left(P_{\mathbf{D}_{\omega}} P_{\mathbf{C}_{\omega}}\right)^{k}(\mathbf{y})-P_{\mathbf{C}_{\omega} \cap \mathbf{D}_{\omega}}(\mathbf{y})\right\| \geq a_{k}
$$


for all $k=1,2, \ldots$. This shows that the sequence $\left\{T_{\omega}^{k}\right\}_{k=1}^{\infty}$ converges arbitrarily slowly to $P_{M}$ as $k \rightarrow \infty$, as asserted.

Theorem 5.3 (Super-polynomial Rate). Let $\omega \in \Omega_{r}$ and assume that the set $A_{\omega}\left(\mathbf{C}_{\omega}^{\perp}\right)$ is not closed in $\mathcal{H}$. Then the sequence $\left\{T_{\omega}^{k}\right\}_{k=1}^{\infty}$ converges super-polynomially fast to $P_{M}$, as $k \rightarrow \infty$, on some dense linear subspace $Y_{\omega} \subset \mathcal{H}$.

Proof. The argument follows the proof of [33, Theorem 14]. In view of Theorem 5.1, the subspace $\mathbf{C}_{\omega}^{\perp}+\mathbf{D}_{\omega}^{\perp}$ is not closed. By Theorem 1.3 applied to $\mathbf{C}_{\omega}$ and $\mathbf{D}_{\omega}$, there is a dense linear subspace $\mathbf{X}_{\omega}$ of $\mathbf{H}_{\omega}$ on which the sequence $\left\{\left(P_{\mathbf{C}_{\omega}} P_{\mathbf{D}_{\omega}}\right)^{k}\right\}_{k=1}^{\infty}$ converges super-polynomially fast to $P_{\mathbf{C}_{\omega} \cap \mathbf{D}_{\omega}}$. Define

$$
\mathbf{Y}_{\omega}:=P_{\mathbf{D}_{\omega}}\left(\mathbf{X}_{\omega}\right) \quad \text { and } \quad Y_{\omega}:=\left\{y \in \mathcal{H}: \mathbf{y}=\{y\}_{i=1}^{r} \in \mathbf{Y}_{\omega}\right\}
$$

Note that the linearity of $P_{\mathbf{D}_{\omega}}$ implies that $\mathbf{Y}_{\omega}$ and $Y_{\omega}$ are both linear subspaces of $\mathbf{H}_{\omega}$ and $\mathcal{H}$, respectively. Let $y \in Y_{\omega}$ and $\mathbf{x} \in \mathbf{X}_{\omega}$ be such that $\mathbf{y}=\{y\}_{i=1}^{r}=P_{\mathbf{D}_{\omega}}(\mathbf{x})$. Then, by Lemma 3.1, (3.16) and by the nonexpansivity of $P_{\mathbf{D}_{\omega}}$, for each $n=1,2, \ldots$, we have

$$
\begin{aligned}
k^{n}\left\|T_{\omega}^{k}(y)-P_{M}(y)\right\| & =k^{n}\left\|\left(P_{\mathbf{D}_{\omega}} P_{\mathbf{C}_{\omega}}\right)^{k}(\mathbf{y})-P_{\mathbf{C}_{\omega} \cap \mathbf{D}_{\omega}}(\mathbf{y})\right\|_{\omega} \\
& =k^{n}\left\|P_{\mathbf{D}_{\omega}}\left(P_{\mathbf{C}_{\omega}} P_{\mathbf{D}_{\omega}}\right)^{k}(\mathbf{x})-P_{\mathbf{D}_{\omega}} P_{\mathbf{C}_{\omega} \cap \mathbf{D}_{\omega}}(\mathbf{x})\right\|_{\omega} \\
& \leq k^{n}\left\|\left(P_{\mathbf{C}_{\omega}} P_{\mathbf{D}_{\omega}}\right)^{k}(\mathbf{x})-P_{\mathbf{C}_{\omega} \cap \mathbf{D}_{\omega}}(\mathbf{x})\right\|_{\omega} \rightarrow 0
\end{aligned}
$$

as $k \rightarrow \infty$. This shows that the sequence $\left\{T_{\omega}^{k}\right\}_{k=0}^{\infty}$ converges super-polynomially fast to $P_{M}$ on $Y_{\omega}$.

We now show that $Y_{\omega}$ is a dense linear subspace of $\mathcal{H}$. Indeed, let $x \in \mathcal{H}$ and let $\mathbf{x}:=\{x\}_{i=1}^{r} \in \mathbf{D}_{\omega}$. Since $\mathbf{X}_{\omega}$ is dense in $\mathbf{H}_{\omega}$, there is $\left\{\mathbf{x}_{k}\right\}_{k=1}^{\infty}$ in $\mathbf{X}_{\omega}$ such that $\mathbf{x}_{k} \rightarrow \mathbf{x}$. Let $\mathbf{y}_{k}:=P_{\mathbf{D}_{\omega}}\left(\mathbf{x}_{k}\right)$. Since $\mathbf{y}_{k} \in \mathbf{Y}_{\omega}$, there is $y_{k} \in Y_{\omega}$ such that $\mathbf{y}_{k}=\left\{y_{k}\right\}_{i=1}^{r}$. Again, by Lemma 3.1 and by the nonexpansivity of $P_{\mathbf{D}_{\omega}}$, we arrive at

$$
\left\|y_{k}-x\right\|=\left\|\mathbf{y}_{k}-\mathbf{x}\right\|_{\omega}=\left\|P_{\mathbf{D}_{\omega}}\left(\mathbf{x}_{k}\right)-P_{\mathbf{D}_{\omega}}(\mathbf{x})\right\|_{\omega} \leq\left\|\mathbf{x}_{k}-\mathbf{x}\right\|_{\omega} \rightarrow 0
$$

as $k \rightarrow \infty$. This completes the proof.

Theorem 5.4 (Polynomial Rate). Let $\omega \in \Omega_{r}$. Assume that $y \in A_{\omega}\left(C_{\omega}^{\perp}\right)$. Then there is $C_{\omega}(y)>0$ such that for all $k$, we have

$$
\left\|T_{\omega}^{k}(y)-P_{M}(y)\right\| \leq \frac{C_{\omega}(y)}{\sqrt{k}} .
$$

Proof. We first show that in spite of the possible inequality $\mathbf{C}_{\omega} \cap \mathbf{D}_{\omega} \neq\{\mathbf{0}\}$ (see Theorem 1.4), for all $\mathbf{x}=\left\{x_{i}\right\}_{i=1}^{r} \in \mathbf{C}_{\omega}^{\perp}+\mathbf{D}_{\omega}^{\perp}$ there is $C_{\omega}(\mathbf{x})>0$ such that

$$
\left\|\left(P_{\mathbf{D}_{\omega}} P_{\mathbf{C}_{\omega}}\right)^{k}(\mathbf{x})-P_{\mathbf{C}_{\omega} \cap \mathbf{D}_{\omega}}(\mathbf{x})\right\|_{\omega} \leq \frac{C_{\omega}(\mathbf{x})}{\sqrt{k}}
$$

for all $k=1,2, \ldots$. To this end, let $\mathbf{M}_{1}:=\mathbf{C}_{\omega} \cap\left(\mathbf{C}_{\omega} \cap \mathbf{D}_{\omega}\right)^{\perp}$ and $\mathbf{M}_{2}:=\mathbf{D}_{\omega} \cap\left(\mathbf{C}_{\omega} \cap \mathbf{D}_{\omega}\right)^{\perp}$. Recall again that the projections $P_{\mathbf{C}_{\omega}}$ and $P_{\mathbf{D}_{\omega}}$ commute with $P_{\mathbf{C}_{\omega} \cap \mathbf{D}_{\omega}}$; see (3.16). Similarly to (4.2), they commute with $P_{\left(\mathbf{C}_{\omega} \cap \mathbf{D}_{\omega}\right)^{\perp}}$, that is,

$$
\begin{aligned}
& P_{\mathbf{M}_{1}}=P_{\left(\mathbf{C}_{\omega} \cap \mathbf{D}_{\omega}\right)^{\perp}} P_{\mathbf{C}_{\omega}}=P_{\mathbf{C}_{\omega}} P_{\left(\mathbf{C}_{\omega} \cap \mathbf{D}_{\omega}\right)^{\perp}}, \\
& P_{\mathbf{M}_{2}}=P_{\left(\mathbf{C}_{\omega} \cap \mathbf{D}_{\omega}\right)^{\perp}} P_{\mathbf{D}_{\omega}}=P_{\mathbf{D}_{\omega}} P_{\left(\mathbf{C}_{\omega} \cap \mathbf{D}_{\omega}\right)^{\perp}} .
\end{aligned}
$$


Using their linearity and the above-mentioned commuting properties, we obtain

$$
\begin{aligned}
\left(P_{\mathbf{D}_{\omega}} P_{\mathbf{C}_{\omega}}\right)^{k}-P_{\mathbf{C}_{\omega} \cap \mathbf{D}_{\omega}} & =\left(P_{\mathbf{D}_{\omega}} P_{\mathbf{C}_{\omega}}\right)^{k}-\left(P_{\mathbf{D}_{\omega}} P_{\mathbf{C}_{\omega}}\right)^{k} P_{\mathbf{C}_{\omega} \cap \mathbf{D}_{\omega}} \\
& =\left(P_{\mathbf{D}_{\omega}} P_{\mathbf{C}_{\omega}}\right)^{k} P_{\left(\mathbf{C}_{\omega} \cap \mathbf{D}_{\omega}\right)^{\perp}} \\
& =\left(P_{\mathbf{D}_{\omega}} P_{\mathbf{C}_{\omega}}\right)^{k}\left(P_{\left(\mathbf{C}_{\omega} \cap \mathbf{D}_{\omega}\right)^{\perp}}\right)^{2 k} \\
& =\left(P_{\mathbf{M}_{2}} P_{\mathbf{M}_{1}}\right)^{k} .
\end{aligned}
$$

We may now apply Theorem 1.4 to $\mathbf{M}_{1}$ and $\mathbf{M}_{2}$ because $\mathbf{M}_{1} \cap \mathbf{M}_{2}=\{\mathbf{0}\}$. Thus, for every $\mathbf{x} \in \mathbf{M}_{1}^{\perp}+\mathbf{M}_{2}^{\perp}$ there is $C_{\omega}(\mathbf{x})>0$ such that

$$
\left\|\left(P_{\mathbf{D}_{\omega}} P_{\mathbf{C}_{\omega}}\right)^{k}(\mathbf{x})-P_{\mathbf{C}_{\omega} \cap \mathbf{D}_{\omega}}(\mathbf{x})\right\|_{\omega}=\left\|\left(P_{\mathbf{M}_{2}} P_{\mathbf{M}_{1}}\right)^{k}(\mathbf{x})\right\|_{\omega} \leq \frac{C_{\omega}(\mathbf{x})}{\sqrt{k}} .
$$

Note that, by [17, Theorem 4.6 (5)] (or by (2.24) with $r=2$ ), we obtain

$$
\mathbf{C}_{\omega}^{\perp} \subset \overline{\mathbf{C}_{\omega}^{\perp}+\left(\mathbf{C}_{\omega} \cap \mathbf{D}_{\omega}\right)}=\mathbf{M}_{1}^{\perp} \quad \text { and } \quad \mathbf{D}_{\omega}^{\perp} \subset \overline{\mathbf{D}_{\omega}^{\perp}+\left(\mathbf{C}_{\omega} \cap \mathbf{D}_{\omega}\right)}=\mathbf{M}_{2}^{\perp} .
$$

Consequently, $\mathbf{C}_{\omega}^{\perp}+\mathbf{D}_{\omega}^{\perp} \subset \mathbf{M}_{1}^{\perp}+\mathbf{M}_{2}^{\perp}$, which, when combined with (5.13), proves (5.9).

We may now return to (5.8). Let $y \in A_{\omega}\left(\mathbf{C}_{\omega}^{\perp}\right)$ and let $\mathbf{y}=\{y\}_{i=1}^{r}$. Then $y=A_{\omega}(\mathbf{x})$ for some $\mathbf{x} \in \mathbf{C}_{\omega}^{\perp}$ and, by (3.2), $\mathbf{y}=P_{\mathbf{D}_{\omega}}(\mathbf{x})=\mathbf{x}-P_{\mathbf{D}_{\omega}^{\perp}}(\mathbf{x}) \in \mathbf{C}_{\omega}^{\perp}+\mathbf{D}_{\omega}^{\perp}$. Consequently, by (5.9), there is $C_{\omega}(y):=C_{\omega}(\mathbf{y})>0$ such that

$$
\left\|T_{\omega}^{k}(y)-P_{M}(y)\right\|=\left\|\left(P_{\mathbf{D}_{\omega}} P_{\mathbf{C}_{\omega}}\right)^{k}(\mathbf{y})-P_{\mathbf{C}_{\omega} \cap \mathbf{D}_{\omega}}(\mathbf{y})\right\|_{\omega} \leq \frac{C_{\omega}(y)}{\sqrt{k}}
$$

for all $k=1,2, \ldots$, where the equality follows from (3.10).

\section{Appendix}

It is well known that if a series $\sum_{i=1}^{\infty} y_{i}$ in $\mathcal{H}$ is absolutely convergent, that is, when $\sum_{i=1}^{\infty}\left\|y_{i}\right\|<\infty$, then it is also unconditionally convergent, that is, $\sum_{i=1}^{\infty} y_{\sigma(i)}$ exists for all bijections $\sigma$ in $\mathbb{Z}_{+}$and equals $\sum_{i=1}^{\infty} y_{i}$. At this point recall that the unconditionally convergent series coincide with the absolutely convergent series if and only if the space $\mathcal{H}$ is of finite dimension; see [21].

We slightly strengthen the unconditional convergence in Lemma 6.1 below. To this end, for an absolutely convergent series $\sum_{i=1}^{\infty} y_{i}$ and for any subset $I=\left\{i_{1}, i_{2}, \ldots\right\}$ of $\mathbb{Z}_{+}$, we formally define $\sum_{i \in I} y_{i}:=\sum_{l=1}^{\# I} y_{i_{l}}$. A result similar to Lemma 6.1 can be found, for example, in [26, Theorem 6.3.1] for $\mathcal{H}=\mathbb{R}$.

Lemma 6.1 (Rearrangement Lemma). Let $q \in \mathbb{Z}_{+} \cup\{\infty\}$ and let $\left\{I_{j}\right\}_{j=1}^{q}$ consist of nonempty and pairwise disjoint subsets of $\mathbb{Z}_{+}$, possibly infinite, such that $\mathbb{Z}_{+}=\bigcup_{j=1}^{q} I_{j}$. Assume that the series $\sum_{i=1}^{\infty} y_{i}$ is absolutely convergent. Then

$$
\sum_{i=1}^{\infty} y_{i}=\sum_{j=1}^{q}\left(\sum_{i \in I_{j}} y_{i}\right),
$$

where the summation over $j$, as well as the summations over $i \in I_{j}$, do not depend on the order of summands. 
Proof. Note that the absolute convergence of the series $\sum_{i=1}^{\infty} y_{i}$, when combined with the triangle inequality, leads to

$$
\sum_{j=1}^{q}\left\|\sum_{i \in I_{j}} y_{i}\right\| \leq \sum_{j=1}^{q}\left(\sum_{i \in I_{j}}\left\|y_{i}\right\|\right)=\sum_{i=1}^{\infty}\left\|y_{i}\right\|<\infty,
$$

where the equality holds by [26, Theorem 6.3.1]. Consequently, the series $\sum_{i \in I_{j}} y_{i}$ converges absolutely, hence unconditionally, to some $z_{j} \in \mathcal{H}, j=1, \ldots, q$. Furthermore, the series $\sum_{j=1}^{q} z_{j}$ converges absolutely, hence unconditionally.

Let now $I=\left\{i_{1}, i_{2}, \ldots\right\}, J=\left\{j_{1}, j_{2}, \ldots\right\}$ and $K=I \cup J=\left\{k_{1}, k_{2}, \ldots\right\}$ be countably infinite and increasingly ordered sets of $\mathbb{Z}_{+}$such that $I \cap J=\emptyset$. We claim that

$$
\sum_{k \in K} y_{k}=\sum_{i \in I} y_{i}+\sum_{j \in J} y_{j}
$$

To see this, first define

$$
[n]:=\min \left\{m:\left\{i_{1}, \ldots, i_{n}\right\} \cup\left\{j_{1}, \ldots, j_{n}\right\} \subset\left\{k_{1}, \ldots, k_{m}\right\}\right\}
$$

and

$$
M_{n}:=\left\{\left\{k_{1}, \ldots, k_{[n]}\right\} \backslash\left(\left\{i_{1}, \ldots, i_{n}\right\} \cup\left\{j_{1}, \ldots, j_{n}\right\}\right)\right\} .
$$

Observe that $\min M_{n} \geq n$ whenever the set $M_{n} \neq \emptyset$. Since all the three series in (6.3) converge, we have

$$
\left\|\sum_{k \in K} y_{k}-\sum_{i \in I} y_{i}-\sum_{j \in J} y_{j}\right\|=\lim _{n \rightarrow \infty}\left\|\sum_{l=1}^{[n]} y_{k_{l}}-\sum_{l=1}^{n} y_{i_{l}}-\sum_{l=1}^{n} y_{j_{l}}\right\| \leq \lim _{n \rightarrow \infty} \sum_{i=n}^{\infty}\left\|y_{i}\right\|=0 .
$$

Obviously, formula (6.3) holds when either one, or both, of $I$ and $J$ are finite.

By induction, equality (6.3) carries over to any finite number of sets. In particular, this proves (6.1) for all finite $q \in \mathbb{Z}_{+}$. We now show that (6.1) also holds for $q=\infty$. Indeed, redefine

$$
[n]:=\min \left\{m:\{1, \ldots, n\} \subset I_{1} \cup \ldots \cup I_{m}\right\}
$$

and

$$
M_{n}:=Z_{+} \backslash K_{n}, \quad \text { where } \quad K_{n}:=I_{1} \cup \ldots \cup I_{[n]} .
$$

Note here that since $q=\infty$, we get $M_{n} \neq \emptyset$ and thus $\min M_{n} \geq n$. Consequently, by (6.3) applied to a finite number of sets, first to $K=K_{n}$ and then to $K=\mathbb{Z}_{+}$, we obtain

$$
\left\|\sum_{i=1}^{\infty} y_{i}-\sum_{j=1}^{[n]} z_{j}\right\|=\left\|\sum_{i=1}^{\infty} y_{i}-\sum_{k \in K_{n}} y_{k}\right\|=\left\|\sum_{i \in \mathbb{Z}_{+} \backslash K_{n}} y_{i}\right\| \leq \sum_{i=n}^{\infty}\left\|y_{i}\right\| \rightarrow 0
$$

as $n \rightarrow \infty$.

Acknowledgements. We are grateful to two anonymous referees for all their comments and remarks which helped us improve our manuscript. This work was partially supported by the Israel Science Foundation (Grants 389/12 and 820/17), the Fund for the Promotion of Research at the Technion and by the Technion General Research Fund. 


\section{References}

[1] F. J. Aragón Artacho and R. Campoy, Optimal rates of linear convergence of the averaged alternating modified reflections method for two subspaces, Numer. Algorithms, 82 (2019), pp. 397-421.

[2] N. Aronszajn, Theory of reproducing kernels, Trans. Amer. Math. Soc., 68 (1950), pp. 337-404.

[3] C. Badea, S. Grivaux, And V. Müller, The rate of convergence in the method of alternating projections, Algebra i Analiz, 23 (2011), pp. 1-30.

[4] C. Badea And D. Seifert, Ritt operators and convergence in the method of alternating projections, J. Approx. Theory, 205 (2016), pp. 133-148.

[5] C. Badea And D. SeIfert, Quantified asymptotic behaviour of Banach space operators and applications to iterative projection methods, Pure Appl. Funct. Anal., 2 (2017), pp. 585-598.

[6] H. H. Bauschke, J. Y. Bello Cruz, T. T. A. Nghia, H. M. Phan, and X. Wang, The rate of linear convergence of the Douglas-Rachford algorithm for subspaces is the cosine of the Friedrichs angle, J. Approx. Theory, 185 (2014), pp. 63-79.

[7] H. H. Bauschke, J. Y. Bello Cruz, T. T. A. Nghia, H. M. Phan, and X. Wang, Optimal rates of linear convergence of relaxed alternating projections and generalized Douglas-Rachford methods for two subspaces, Numer. Algorithms, 73 (2016), pp. 33-76.

[8] H. H. BAuschke AND J. M. BorWEIn, On the convergence of von Neumann's alternating projection algorithm for two sets, Set-Valued Anal., 1 (1993), pp. 185-212.

[9] H. H. Bauschke And J. M. Bonwein, On projection algorithms for solving convex feasibility problems, SIAM Rev., 38 (1996), pp. 367-426.

[10] H. H. Bauschke, F. Deutsch, And H. Hundal, Characterizing arbitrarily slow convergence in the method of alternating projections, Int. Trans. Oper. Res., 16 (2009), pp. 413-425.

[11] H. H. Bauschke, D. Noll, And H. M. Phan, Linear and strong convergence of algorithms involving averaged nonexpansive operators, J. Math. Anal. Appl., 421 (2015), pp. 1-20.

[12] P. A. Borodin ANd E. KopeckÁ, Alternating projections, remotest projections, and greedy approximation, J. Approx. Theory, 260 (2020), 105486, 16 pp.

[13] J. M. Borwein, G. LI, And M. K. TAM, Convergence rate analysis for averaged fixed point iterations in common fixed point problems, SIAM J. Optim., 27 (2017), pp. 1-33.

[14] A. Cegrelski, Iterative methods for fixed point problems in Hilbert spaces, vol. 2057 of Lecture Notes in Mathematics, Springer, Heidelberg, 2012.

[15] A. Cegielski, S. Reich, And R. Zalas, Regular sequences of quasi-nonexpansive operators and their applications, SIAM J. Optim., 28 (2018), pp. 1508-1532.

[16] F. Deutsch, Rate of convergence of the method of alternating projections, in Parametric optimization and approximation (Oberwolfach, 1983), vol. 72 of Internat. Schriftenreihe Numer. Math., Birkhäuser, Basel, 1985, pp. 96-107.

[17] F. Deutsch, Best approximation in inner product spaces, vol. 7 of CMS Books in Mathematics/Ouvrages de Mathématiques de la SMC, Springer-Verlag, New York, 2001.

[18] F. Deutsch And H. Hundal, Slow convergence of sequences of linear operators II: arbitrarily slow convergence, J. Approx. Theory, 162 (2010), pp. 1717-1738. 
[19] F. Deutsch And H. Hundal, Arbitrarily slow convergence of sequences of linear operators: a survey, in Fixed-point algorithms for inverse problems in science and engineering, vol. 49 of Springer Optim. Appl., Springer, New York, 2011, pp. 213-242.

[20] F. Deutsch and H. Hundal, Arbitrarily slow convergence of sequences of linear operators, in Infinite products of operators and their applications, vol. 636 of Contemp. Math., Amer. Math. Soc., Providence, RI, 2015, pp. 93-120.

[21] A. Dvoretzky and C. A. Rogers, Absolute and unconditional convergence in normed linear spaces, Proc. Nat. Acad. Sci. U.S.A., 36 (1950), pp. 192-197.

[22] I. Halperin, The product of projection operators, Acta Sci. Math. (Szeged), 23 (1962), pp. 96-99.

[23] R. B. Holmes, Geometric functional analysis and its applications, Springer-Verlag, New York-Heidelberg, 1975. Graduate Texts in Mathematics, No. 24.

[24] M. M. IsRael, JR. AND S. ReICH, Extension and selection problems for nonlinear semigroups in Banach spaces, Math. Japon., 28 (1983), pp. 1-8.

[25] S. Kayalar and H. L. Weinert, Error bounds for the method of alternating projections, Math. Control Signals Systems, 1 (1988), pp. 43-59.

[26] I. KRIZ AND A. PUltr, Introduction to mathematical analysis, Birkhäuser/Springer, Basel, 2013.

[27] M. L. LAPIDus, Generalization of the Trotter-Lie formula, Integral Equations Operator Theory, 4 (1981), pp. 366-415.

[28] U. Mosco, Convergence of convex sets and of solutions of variational inequalities, Advances in Math., 3 (1969), pp. 510-585.

[29] G. PIERrA, Decomposition through formalization in a product space, Math. Programming, 28 (1984), pp. 96115.

[30] E. Pustylnik, S. Reich, And A. J. Zaslavski, Convergence of non-periodic infinite products of orthogonal projections and nonexpansive operators in Hilbert space, J. Approx. Theory, 164 (2012), pp. 611-624.

[31] E. Pustylnik, S. Reich, And A. J. Zaslavski, Inner inclination of subspaces and infinite products of orthogonal projections, J. Nonlinear Convex Anal., 14 (2013), pp. 423-436.

[32] S. REICH, A limit theorem for projections, Linear and Multilinear Algebra, 13 (1983), pp. 281-290.

[33] S. Reich And R. ZAlas, The optimal error bound for the method of simultaneous projections, J. Approx. Theory, 223 (2017), pp. 96-107.

[34] M. K. TAM, Gearhart-Koshy acceleration for affine subspaces, 2020, https://arxiv.org/abs/2007.00844.

[35] M. TsukadA, Convergence of best approximations in a smooth Banach space, J. Approx. Theory, 40 (1984), pp. 301-309.

[36] J. von Neumann, On rings of operators. Reduction theory, Ann. of Math. (2), 50 (1949), pp. 401-485. 